\title{
Extensive Plasmid Library to Prepare Tau Protein Variants and Study Their Functional Biochemistry
}

Thomas K. Karikari,* Sophie Keeling, Emily Hill, Juan Lantero Rodríguez, David A. Nagel, Bruno Becker, Kina Höglund, Henrik Zetterberg, Kaj Blennow, Eric J. Hill, and Kevin G. Moffat

Cite This: ACS Chem. Neurosci. 2020, 11, 3117-3129

Read Online

\section{ACCESS \\ Џlll Metrics \& More \\ Article Recommendations \\ Supporting Information}

ABSTRACT: Tau neurofibrillary tangles are key pathological features of Alzheimer's disease and other tauopathies. Recombinant protein technology is vital for studying the structure and function of tau in physiology and aggregation in pathophysiology. However, open-source and well-characterized plasmids for efficiently expressing and purifying different tau variants are lacking. We generated 44 sequence-verified plasmids including those encoding full length (FL) tau-441, its fourrepeat microtubule-binding (K18) fragment, and their respective selected familial pathological variants (N279K, V337M, P301L, C291R, and S356T). Moreover, plasmids for expressing single (C291A), double (C291A/C322A), and triple (C291A/C322A/ I260C) cysteine-modified variants were generated to study alterations

Plasmids for expressing recombinant tau


in cysteine content and locations. Furthermore, protocols for producing representative tau forms were developed. We produced and characterized the aggregation behavior of the triple cysteine-modified tau-K18, often used in real-time cell internalization and aggregation studies because it can be fluorescently labeled on a cysteine outside the microtubule-binding core. Similar to the wild type (WT), triple cysteine-modified tau-K18 aggregated by progressive $\beta$-sheet enrichment, albeit at a slower rate. On prolonged incubation, cysteine-modified K18 formed paired helical filaments similar to those in Alzheimer's disease, sharing morphological phenotypes with WT tau-K18 filaments. Nonetheless, cysteine-modified tau-K18 filaments were significantly shorter $(p=0.002)$ and mostly wider than WT filaments, explainable by their different principal filament elongation pathways: vertical (end-to-end) and lateral growth for WT and cysteine-modified, respectively. Cysteine rearrangement may therefore induce filament polymorphism. Together, the plasmid library, the protein production methods, and the new insights into cysteine-dependent aggregation should facilitate further studies and the design of antiaggregation agents.

KEYWORDS: Tau, plasmid, MAPT mutations, Alzheimer's disease, frontotemporal dementia, expression and purification

\section{INTRODUCTION}

The microtubule (MT) associated protein tau (MAPT) gene, which encodes the tau protein, is located on chromosome 17q21. ${ }^{1,2}$ MAPT comprises 16 exons some of which are alternatively spliced to generate tau isoforms that are differentially expressed in tissues and in development. ${ }^{3,4}$ The adult human brain has six tau isoforms produced from the alternative splicing of exons 2,3 , and 10. ${ }^{1,5}$ Tau is comprised of a number of regions: the $\mathrm{N}$-terminal projection domain, proline-rich domains in the midregion, and the C-terminal assembly domain, the latter containing the MT binding region [MTBR] and the C-terminus, separated by two proline-rich regions. ${ }^{1,5}$ The isoforms can be distinguished by the number of alternatively spliced inserts at the N-terminus $(0,1$, or $2 \mathrm{~N}$ terminus inserts) and in the MTBR (3 or 4 MTBR repeats). ${ }^{1,5}$ In normal adult humans, a $1: 1$ ratio of $4 \mathrm{R}$ to $3 \mathrm{R}$ is maintained. ${ }^{6}$ However, this isoform balance is disturbed in pathophysiological conditions, with tauopathies biochemically categorized based on their tau isoform composition. Primary tauopathies (including corticobasal degeneration, progressive supranuclear palsy, and globular glial tauopathy) tend to have $4 \mathrm{R}$ tau isoforms (except Pick's disease with $3 \mathrm{R}$ tau), while secondary tauopathies such as Alzheimer's disease $(\mathrm{AD})$ and chronic traumatic encephalopathy have a mix of $3 R$ and $4 R$ isoforms. $^{7-11}$ The alternative splicing of exon 10 has been particularly linked to several tauopathies since it affects the ratio of three-repeat (3R) to four-repeat (4R) tau isoforms. ${ }^{12}$

The most-studied physiological function of tau is its support of axonal transport by binding to and modulating the stability

Received: July 23, 2020

Accepted: August 24, 2020

Published: August 24, 2020 
of MTs. ${ }^{13,14}$ Tau binds to MTs at the $\alpha$ - and $\beta$-tubulin heterodimer interface using specific amino acid sequences, most of which are located within the MTBR. ${ }^{15}$ This generates a local hairpin structure involving the hexapeptide motifs ${ }^{275}$ VQIINK ${ }^{280}$ and ${ }^{306}$ VQIVYK ${ }^{311}$, with numbering corresponding to 441 amino acid-long full-length (FL) tau (Uniprot \#P10636-8). ${ }^{15}$ Over 110 single nucleotide polymorphisms in MAPT have been reported, with more than a dozen associated with specific familial forms of frontotemporal dementia (FTD).$^{16-19}$ Several of these disease-associated mutations can interfere with the protein's MT-binding function, by either potentiating or decreasing this activity. ${ }^{20-22}$ How specific MAPT mutations cause distinct effects on tau's MT interactions are not clearly understood, with further studies needed.

In disease conditions, tau can become hyperphosphorylated on specific residues leading to its detachment from MTs, thereby compromising axonal transport/assembly. ${ }^{23}$ Following this loss of function, the soluble, unfolded phosphorylated tau proteins can self-polymerize to eventually form paired helical filaments (PHFs) and other filamentous aggregates enriched in $\beta$-sheet structures, via oligomer and protofibril intermediates. ${ }^{24}$ These filaments are critical components of neurofibrillary tangles (NFTs), which are hallmarks of $\mathrm{AD}$ and other tauopathies. $^{24,25}$ Tau aggregation can also occur through disulfide interactions involving the native cysteine residues, either at position 291 only ( $3 \mathrm{R}$ isoforms) or at 291 and 322 (4R isoforms). ${ }^{26-28}$ Intermolecular disulfide bonding is thought to promote aggregation, while intramolecular bonding inhibits aggregation. ${ }^{29} 4 \mathrm{R}$ tau isoforms can therefore aggregate faster than 3R tau isoforms, possibly due to their capacity to form increasing polymers of disulfide-bonded aggregates relative to $3 \mathrm{R}$ tau isoforms whose disulfide-dependent aggregation is limited to dimerization. ${ }^{30}$ However, the aggregation-prone ${ }^{275}$ VQIINK ${ }^{280}$ hexapeptide motif in $4 R$, but not 3R, tau isoforms may also contribute to the observed higher aggregation tendency of $4 \mathrm{R}$ tau, although little investigation has focused on this hypothesis. ${ }^{31,32}$

Tau aggregation requires at least one of the hexapatide motifs ${ }^{275}$ VQIINK $^{280}$ and ${ }^{306}$ VQIVYK $^{311}$ within the MTBR $^{31,33,34}$ Notably, a number of familial FTD-associated MAPT mutations, again located in the MTBR (e.g., P301L, V337M, N279K), are known to either accelerate or decrease tau aggregation kinetics and can form filaments of distinct structural phenotypes compared to the wild type (WT) ${ }^{20-22,35}$ Furthermore, some disease-associated mutations demonstrate stark differences in their ability to be "seeded" by filaments from the WT 4R tau MTBR (the K18 fragment), with P301L and P301S exhibiting robust template misfolding capabilities. $^{35,36}$ This suggests that tau aggregation is conformationdependent and that selected MAPT mutations may affect aggregation through distinct mechanisms. Moreover, the temporal and spatial progression of tau aggregate accumulation in $\mathrm{AD}$ and other tauopathies, which correlates well with cognitive decline, is thought to occur as a result of intercellular transfer and seeding of tau aggregates in cells. ${ }^{37,38}$ Specific $M A P T$ mutations can influence this process through distinct effects on exogenous tau internalization and release. ${ }^{35,39}$ Moreover, tau aggregation can be a downstream event in $\mathrm{AD}$ (especially evident in familial $\mathrm{AD}$ due to mutations in the APP and PSEN genes), as well as in some other disorders (e.g., Niemann-Pick type C). ${ }^{40}$ Together, these data demonstrate that aggregation is a critical determinant of the pathophysiological functions of tau.

Much of the insight into the physiological and pathophysiological functions of tau (including MT binding, aggregation, and intercellular propagation) has been obtained using tau from recombinant sources, with many of these processes corroborated using human or animal tissues. ${ }^{2,23,41-45}$ These data establish recombinant technology as a validated strategy for generating functional tau proteins whose properties closely align with those of human brain tau protein. However, recombinant preparation of tau can be challenging and timeconsuming, given that one needs to design and verify gene and plasmid sequences and perform polymerase chain reaction (PCR), restriction digestion, and ligation, before transforming a host of choice for expression. Even though some of these problems can be addressed by plasmid sharing between laboratories, the recipients often have to develop and optimize their own expression and purification protocols. These bottlenecks can unduly delay the preparation of recombinant tau. In an attempt to help address these shortcomings, we have generated a library of 44 sequence-verified plasmids for expressing FL human tau-441 (the longest tau isoform in the adult human brain) and truncated tau consisting of the $4 \mathrm{R}$ MTBR fragment (K18). These include plasmids for producing specific FTD variants of FL and K18 tau (V337M, N279K, P301L, C291R, and S356T), as well as cysteine-modified variants (C291A, C291A/C322A, C291A/C322A/I260C) of the FL and K18 fragment of WT and FTD tau variants.

Since FL 4R tau contains two native cysteine residues, ${ }^{28}$ single and double cysteine modifications (usually replaced by alanine or serine) allow for studies of contributions of one or both native cysteine residues to the protein's properties of interest (MT binding, stabilization, and polymerization; ${ }^{46,47}$ filamentous aggregation and associated conformational changes $^{26,28,48-52}$ ). For studies aimed at investigating incipient tau aggregation and the intercellular propagation and toxicity of extracellular tau using real-time and/or kinetic assays, maleimide-based fluorophore labeling is, however, often used. This technique, first introduced by the Mandelkow groups, ${ }^{53,54}$ often involves triple cysteine modification where the native cysteine residues are removed (C291A/C322A) and a new cysteine residue introduced outside the central core of the MTBR (I260C) to minimize or avoid steric hindrance. $^{35,49,53-58}$ Notwithstanding its increasing popularity, it has not been well studied if this triple cysteine modification affects tau aggregation kinetics, filament formation, and secondary structure. Importantly, answering this question will help provide a validated context to interpreting outcomes of experiments using these constructs. We therefore took advantage of the plasmid resources and the expression and purification methods developed to generate and carefully analyze the aggregation and Alzheimer-like filament formation paths and products of K18 C291A/C322A/I260C and K18 WT.

\section{RESULTS AND DISCUSSION}

Construction and Verification of Plasmids for Expressing FL and Truncated Tau and Their Respective Selected FTD Variants. pProEx-HTa plasmids encoding human WT FL or K18 tau (Figure S1) were created, and their sequences were verified by DNA sequencing. The coding sequences derived from the amino acid sequence for tau 1-441 (Uniprot \#P10636-8) were generated by PCR from template 
pCMV-tau plasmids and cloned into the EheI and EcoRI restriction sites of the pProEx-HTa plasmid as previously described. ${ }^{59}$ DNA sequencing was performed externally by GATC Biotech AG, Konstanz, Germany. Protein expression was controlled by a trc promoter. The plasmid description is available in Table S1. Moreover, each plasmid had a cleavable $\mathrm{N}$-terminal 6xHis tag for purification by immobilized metal affinity chromatography (IMAC; Figure S1). Each K18 plasmid also had a c-Myc tag inserted between the 6xHis tag and the genetic construct of interest, while the FL tau plasmids either had a C-terminus c-Myc tag or an N-terminus FLAG tag downstream of the 6xHis tag (Figure S1 and Table S1).

Specific FTD mutations can alter the physiological (including MT binding and stabilization and subcellular localization $^{21,60-62}$ ) and pathophysiological (e.g., phosphorylation, aggregation pathway, kinetics, and products $^{21,22,35,63-66}$ ) functions of tau. To increase the utility of our genetic resource library, the template WT plasmids were used to generate plasmids for expressing specific FTD variants of FL and K18 tau. We focused on selected FTD mutations spanning the MTBR, including some with relatively well characterized biochemical properties (e.g., V337M, N279K, and $\mathrm{P} 301 \mathrm{~L}^{21,36,65-69}$ ) and others about which little is known (e.g., C291R and S356 $\mathrm{T}^{70,71}$ ). Successful generation of these constructs (Figure 1) was verified by DNA sequencing, with the codon changes and site-directed mutagenesis primers listed in Table S2.

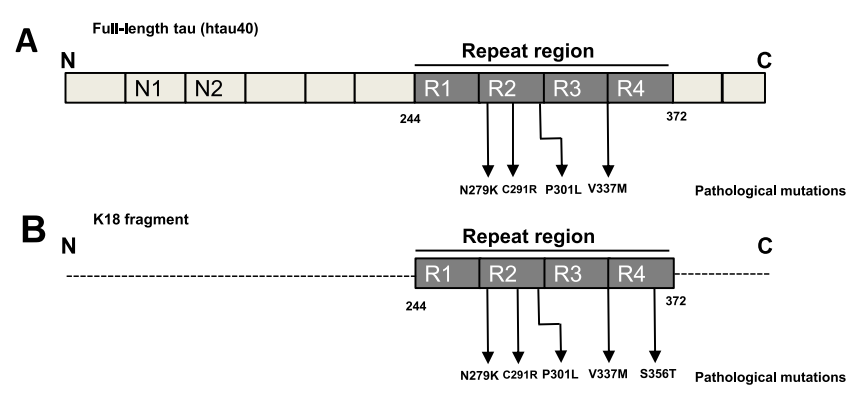

Figure 1. Schematic illustration of FL and truncated tau plasmid constructs carrying specific pathological MAPT mutations generated in this study. (A) In addition to the template plasmid for expressing WT FL tau-441, sequence-verified alternatives each carrying one of the FTD variants N279K, C291R, P301L, and V337M were constructed. The location of each pathological mutation in the MTBR is shown. (B) Plasmid constructs encoding the WT K18 fragment (the MTBR only) and its variants carrying one of the N279K, C291R, P301L, V337M, and S356T mutations created in this study. The location of each mutation is indicated.

Design and Verification of Plasmids for Expressing Cysteine Variants of FL, Truncated, and FTD Tau. The redox state of $4 \mathrm{R}$ tau, which has two native cysteine residues, can critically influence its properties. ${ }^{28,46,47,72}$ To support studies focused on understanding potential effects of the availability, quantity, and location of cysteine residues on tau functions, plasmids for expressing FL and truncated tau (both WT and FTD variants) bearing single (C291A), double (C291A/C322A), or triple (C291A/C322A/I260C) cysteine modifications were further generated (Figure 2 and Table S1). Codon changes made and primers used to generate these variants can be found in Table S3, with DNA sequencing primers in Table S4. Figure 3 shows a schematic illustration of the first (FTD mutations) and second order (cysteine
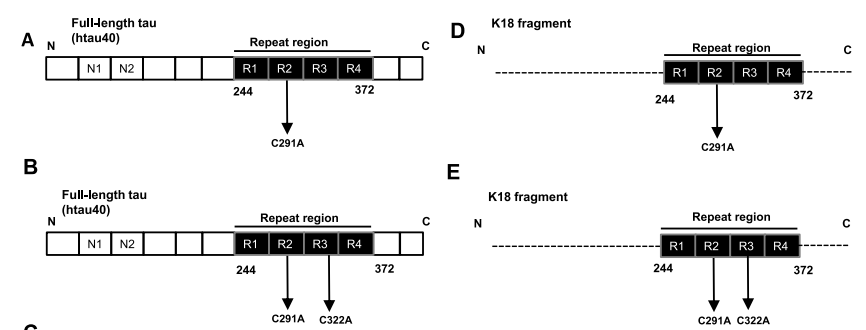

E

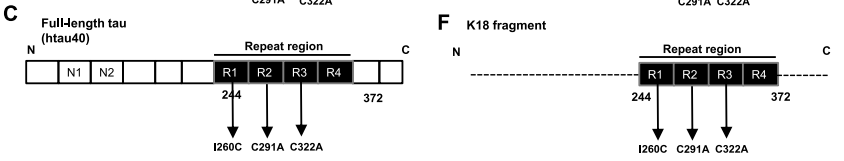

Figure 2. Schematic illustration of plasmid constructs created for the expression of WT and FTD versions of FL and K18 proteins containing specific cysteine modifications. Single (C291A), double (C291A/C322A), and triple (C291A/C322A/I260C) cysteine modifications in FL tau-441 (WT or FTD variants) are illustrated in (A), (B), and (C), respectively. (D), (E), and (F) describe the single, double, and triple cysteine modifications, respectively, in the K18 group of plasmids created in this study. The dashed lines denote the full length tau sequence which is removed in the truncated K18 construct.

modifications) plasmids generated based on each WT template construct.

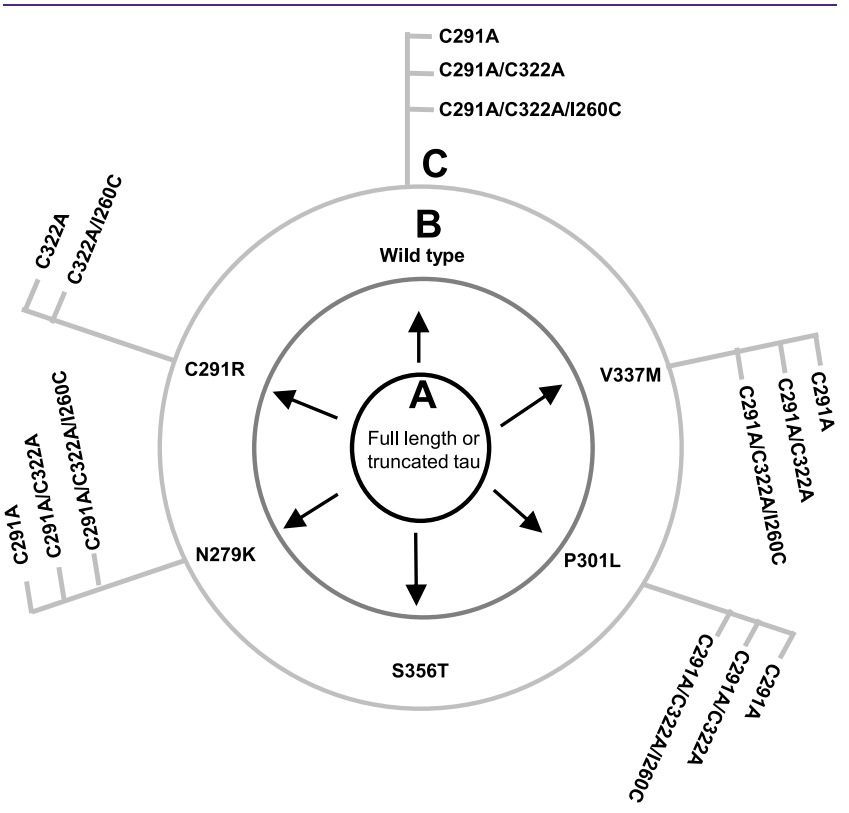

Figure 3. Incremental generation of plasmids expressing tau protein variants carrying specific FTD-associated mutations and cysteine modifications. Template plasmids for expressing WT FL and truncated tau were first created (A). Subsequently, mutagenesis of these base plasmids was used to generate variants encoding specific FTD mutations (B). Next, single, double, or triple cysteine modifications were introduced in the primary (WT) and secondary (FTD variants) plasmids to generate new forms of tau with variable cysteine content $(\mathrm{C})$.

Expression and Purification of WT and Triple Cysteine-Modified FL Tau. To help simplify recombinant tau production, we developed a protocol for expressing and purifying selected constructs from our plasmid library. We adapted a high-yield method ${ }^{59}$ to produce the WT and triple cysteine-modified forms of both FL tau and K18. Plasmids 
transformed into BL21(DE3)*pRosetta Escherichia coli were expressed and purified by immobilized metal affinity chromatography (IMAC), taking advantage of the cleavable N-terminal 6xHis tags, as described (see the Methods section). Expressed protein variants were initially purified by IMAC. Tau-positive fractions, identified after SDS-PAGE and Western blotting, were further purified by size exclusion chromatography (SEC), and the purest fractions were selected (Figure 4).
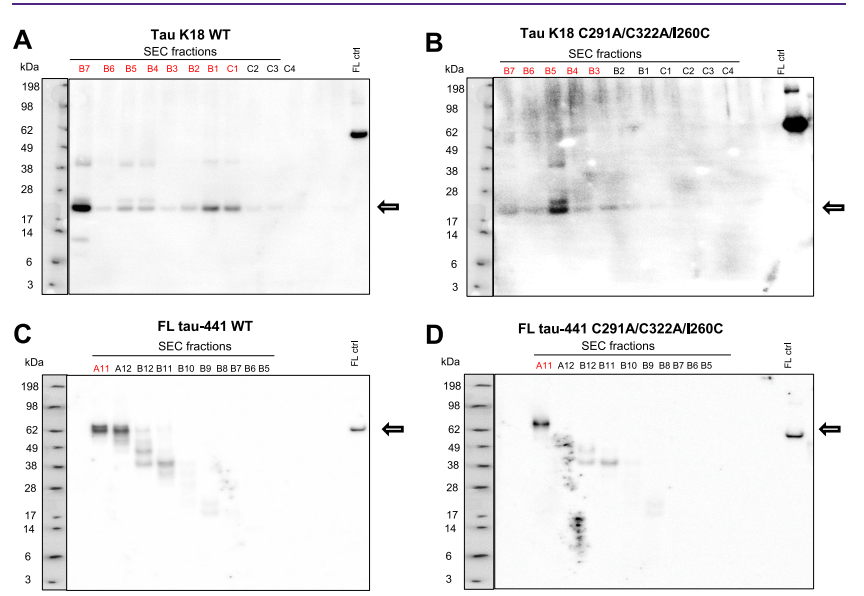

Figure 4. Expression and purification of WT FL and truncated tau K18 and their triple cysteine modified variants. Representative characterization of tau purification by Western blotting. K18 WT (A) and K18 triple cysteine-modified (B) were analyzed by Western blotting using the 77G7 antitau antibody (epitope = aa 316-355), while the WT (C) and triple cysteine modified (D) FL tau were probed with Tau12 (epitope $=$ aa 6-18). A previous preparation of WT recombinant FL tau-441 was used as a control on each blot. The alphanumeric labeling of lanes refers to the analyzed fractions from size exclusion chromatography that were analyzed by Western blotting. Bands corresponding to tau which is in the conformation of interest are indicated by black arrows. Fractions labeled in red were the purest/highest yield selected for further processing. Figures S2 and S3 show Western blot characterization of the initial IMAC-based purification. Size characterization of purified tau forms using SEC can be found in Figure S4.

Cleavage of the $6 \mathrm{xHis}$ tag was achieved by overnight dialysis in the presence of tobacco etch virus (TEV) protease, followed by a second IMAC purification resulting in the $6 \mathrm{xHis}$ tag bound to the column, liberating the cleaved protein as either tau-K18 with a c-Myc tag or FL tau-441 with a FLAG tag. ${ }^{59}$

The IMAC-purified 6xHis-tagged tau K18 fragments migrated on SDS-PAGE gels with electrophoretic mobility corresponding to $\sim 22 \mathrm{kDa}$ and were recognized by the $77 \mathrm{G} 7$ (Figure 4A,B) and K9JA ${ }^{59}$ antitau antibodies both selective for the MTBR (epitopes = amino acids (aa) 316-355 for 77G7 and 244-372 for K9JA). Cleavage of the 6xHis tag was demonstrated by lost reactivity to the anti-His antibody, ${ }^{59}$ reducing the apparent molecular weight of the K18 peptides to $\sim 17 \mathrm{kDa}$ by Western blotting (Figure S2). WT and triple cysteine-modified FL tau were also purified to near homogeneity with apparent sizes of $\sim 62 \mathrm{kDa}$ on SDSPAGE/Western blot, with strong reactivity to the N-terminus antitau antibody Tau12 (epitope = aa 6-18; Figure 4C,D) and the MTBR-binding K9JA (Figure S3). For characterization of purified tau species using SEC, see Figure S4.

Thioflavin T Assay Shows That Triple Cysteine Modification Decreases the Aggregation Propensity of WT Tau K18. A ThT assay was developed to study if the triple cysteine modification affected the aggregation kinetics of K18 WT. K18 WT had an initial mean signal of $\sim 1200$ relative fluorescent units (RFU), which after a lag phase of $\sim 1.5 \mathrm{~h}$, increased sharply until reaching a plateau at $\sim 11,400 \mathrm{RFU}$ just after $5 \mathrm{~h}$ (Figure 5A,B). On the contrary, K18 C291A/C322A/

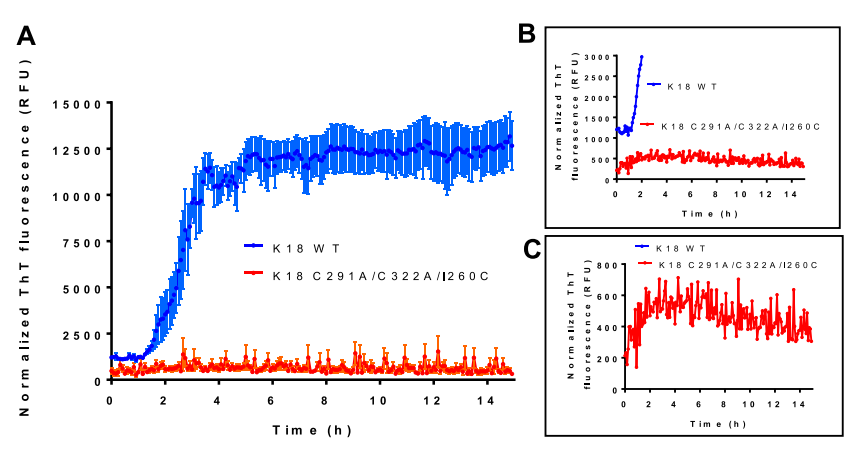

Figure 5. Aggregation kinetics of WT and triple cysteine-modified tau K18 in the ThT fluorescence assay. (A) Aggregation kinetics of the tau K18 variants each normalized to corresponding signals from the constituent buffer only + ThT (negative control). Normalization was achieved by subtracting the ThT signal generated by buffer only at each time point from the signals generated by the tau K18 constructs. Data are expressed as mean \pm SEM, $n=4$ and $n=11$ for K18 WT and K18 C291A/C322A/I260C, respectively. (B) Magnified image focusing on values $\leq 3000 \mathrm{RFU}$ to highlight the shape of the kinetic curve for K18 C291A/C322A/I260C. (C) ThT fluorescence values $\leq$ $800 \mathrm{RFU}$ demonstrate that K18 C291A/C322A/I260C recorded a brief rise in aggregation kinetics which then plateaued.

I260C had severely reduced ThT kinetics: an initial mean signal at $\sim 200$ RFU increased slowly until reaching a peak at $\sim 600 \mathrm{RFU}$ after $3 \mathrm{~h}$ (Figure 5A-C). The kinetic behavior of K18 C291A/C322A/I260C was more visible by focusing on RFU values $<800$, which showed that the peptide had an initial small increase in ThT fluorescence which immediately stabilized after $3 \mathrm{~h}$ : no discernible lag phase was recorded (Figure 5C). The data suggest that the triple cysteine modification decreases the aggregation rate of tau K18 20 fold in nonreducing conditions.

WT Tau K18 Forms Filaments Faster than Its Triple Cysteine-Modified Variant. Co-incubation of amyloidogenic proteins with the ThT dye can lead to unintended effects on aggregation. ${ }^{73}$ To rule out the possibility that the decreased aggregation kinetics of $\mathrm{K} 18 \mathrm{C} 291 \mathrm{~A} / \mathrm{C} 322 \mathrm{~A} / \mathrm{I} 260 \mathrm{C}$ was due to anomalous interactions with $\mathrm{ThT}$, a dye-free aggregation assay was performed. No filament formation was recorded for either sample incubated for $15 \mathrm{~h}$ at $37{ }^{\circ} \mathrm{C}$ without shaking. Consequently, the incubation time was extended to promote filament assembly. After 5 days in the same conditions, K18 WT, but not K18 C291A/C322A/I260C, formed filamentous aggregates (Figure 6A,B). This corroborates the ThTmonitored aggregation data in Figure 5 that the triple cysteine modification decreases the aggregation kinetics of K18 WT in nonreducing conditions.

End Point Tau K18 Aggregates Have Converging and Diverging Structural Phenotypes. Incubation time for aggregation reactions was further increased to 16 days to enable both tau variants to form mature filamentous aggregates. TEM analysis showed that K18 WT and K18 C291A/C322A/I260C formed filaments with both converging and diverging phenotypes. Both variants formed paired helical filaments (PHFs) with varying degrees of twist (Figure 7Ai- 


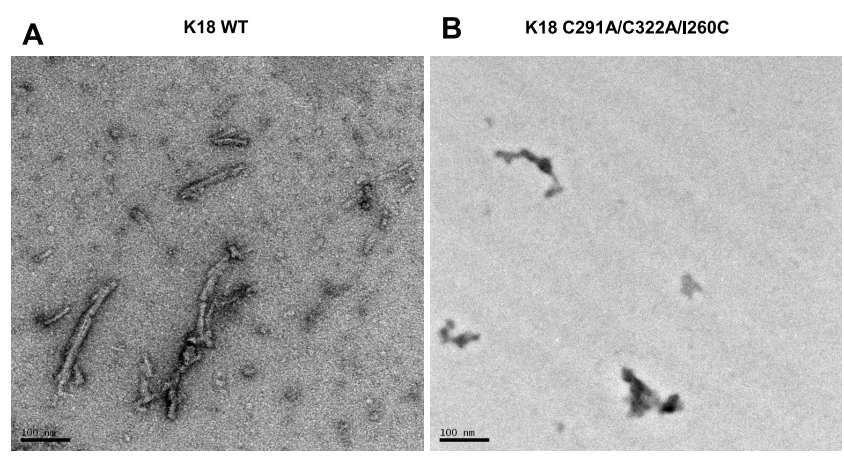

Figure 6. Structural characterization of tau K18 aggregation products in 5-day aggregation reactions. Representative TEM micrographs for tau K18 WT (A) and K18 C291A/C322A/I260C (B). Images were taken using identical TEM settings at $25000 \times$ magnification. Scale bars $=100 \mathrm{~nm}$.

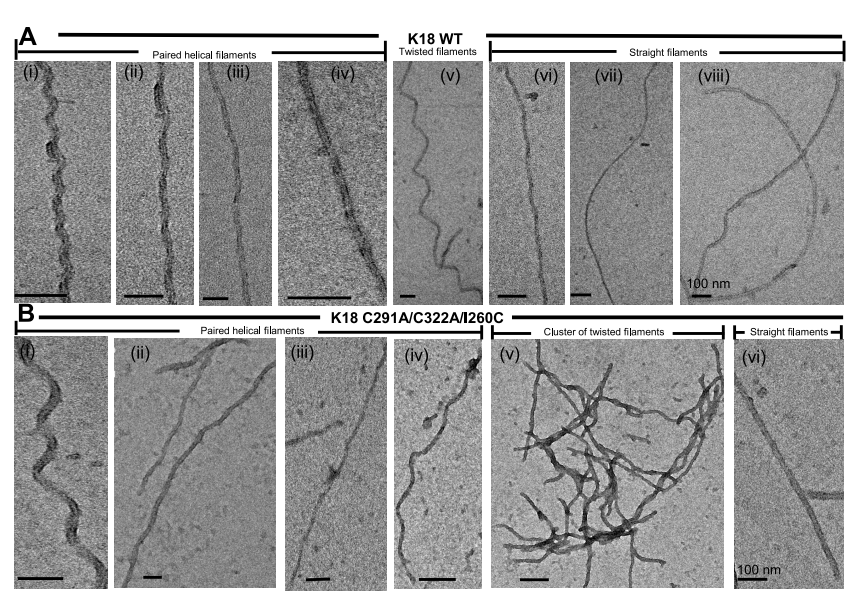

Figure 7. Characterization of the aggregation products of tau K18 variants after 16 days of incubation. Representative TEM micrographs for tau K18 WT (A) and K18 C291A/C322A/I260C (B) are shown, demonstrating both shared and distinct morphological features between filaments formed by the two constructs. Images were taken using identical TEM settings at $12000 \times$ magnification. Scale bars $=$ $100 \mathrm{~nm}$ for each panel.

iv,Bi-iv). In addition to classical PHFs (Figure 7Aiii,iv), K18 WT formed multistriated ribbons (multiple PHFs interwoven together; Figure 8Ai) and ribbons with central depression (multistriated ribbons of uneven widths; Figure 7Aii), consistent with previous observations. ${ }^{74}$ Furthermore, a few twisted filaments lacking the classical PHF arrangement were observed (Figure 7Av). Moreover, in K18 WT preparations, two types of straight (nontwisted) filaments (SFs) were recorded: those with the regular linear appearance (Figure 7Avi) and others with a curved phenotype (Figure 7Avii,viii). K18 C291A/C322A/I260C formed mostly PHFs which often made lateral contacts with others and sometimes formed clusters (Figure $7 \mathrm{Bi}-\mathrm{v}$ ). A few SFs were also observed (Figure $7 \mathrm{Bvi})$.

WT tau K18 filaments were significantly longer than those of K18 C291A/C322A/I260C ( $p=0.002$, two-tailed MannWhitney test; mean lengths for WT and C291A/C322A/ $\mathrm{I} 260 \mathrm{C}=1457.00 \pm 76.33 \mathrm{~nm}$ and $1069.00 \pm 86.73 \mathrm{~nm}$ respectively; Figure 8A). On the contrary, several K18 C291A/ C322A/I260C filaments appeared to be wider than those of the WT (mean width for WT and cysteine-modified $=16.80 \pm$ $1.27 \mathrm{~nm}$ and $20.38 \pm 1.95 \mathrm{~nm}$ ) although this difference was
A
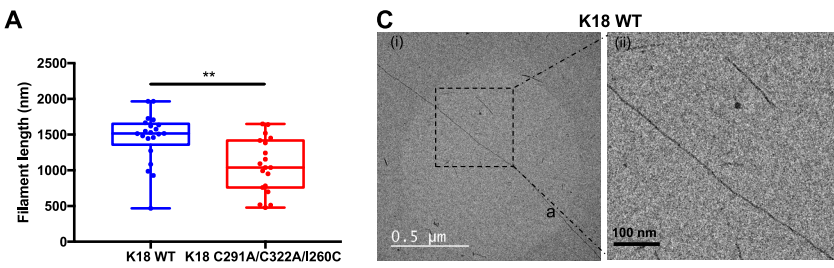

B
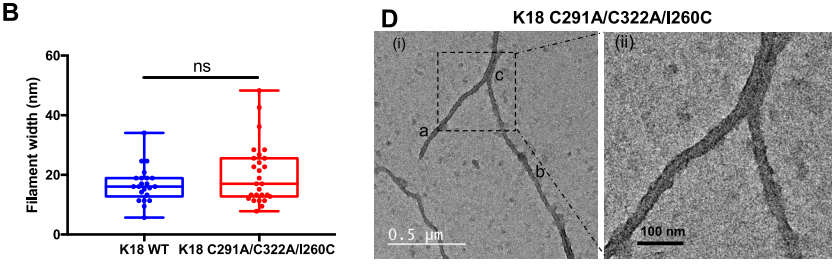

Figure 8. Quantitative characterization of K18 WT and K18 C291A/ C322A/I260C filaments. (A) Box plot of filament lengths for the two tau K18 variants. Two-tailed Mann-Whitney test, $* *=p=0.002$. Mean lengths for WT and cysteine-modified K18 filaments $=1457.00$ $\pm 76.33 \mathrm{~nm}$ and $1069.00 \pm 86.73 \mathrm{~nm}$, respectively. Median lengths for K18 WT and K18 C291A/C322A/I260C = 1515.00 and 1038.00 $\mathrm{nm}$, respectively. The filament length analysis included $n=21$ data points for K18 WT and $n=19$ for K18 C291A/C322A/I260C. (B) Box plot of filament widths for the tau K18 variants. Two-tailed Mann-Whitney test, $p=0.376$; ns $=$ not significant. Mean widths for K18 WT and K18 C291A/C322A/I260C $=16.80 \pm 1.27 \mathrm{~nm}$ and $20.38 \pm 1.95 \mathrm{~nm}$, respectively. Median widths $=16.07$ and $17.05 \mathrm{~nm}$ for K18 WT and K18 C291A/C322A/I260C, respectively. The filament width analysis included $n=22$ data points for K18 WT and $n$ $=28$ for K18 C291A/C322A/I260C. (C) (i) An example TEM image showing the long and slender appearance of the K18 WT filament. The image shown here has a length of $1966.59 \mathrm{~nm}$ and a width of 5.68 $\mathrm{nm}$. Position where the width measurement was taken is marked "a" (ii) A digitally zoomed image highlighting the long and thin appearance of the filament structure shown in (i). (D) (i) A representative TEM micrograph demonstrating lateral connections between two K18 C291A/C322A/I260C filaments. The upper sections of these filaments had coalesced to form a thicker filament. The filament on the left is $1038.08 \mathrm{~nm}$ long and $36.16 \mathrm{~nm}$ wide (position marked "a"), while the one on the right is $1519.06 \mathrm{~nm}$ long and $22.72 \mathrm{~nm}$ wide (position marked "b"). The width at the point of coalescence (marked "c") is $48.30 \mathrm{~nm}$. (ii) Digital zoom showing a magnified view of the lateral interactions observed in (i). The images in $(C)(i)$ and $(D)(i)$ were both acquired using identical TEM settings on the same day at $12000 \times$ magnification.

not found to be significant ( $p=0.376$, two-tailed MannWhitney test; Figure 8B). Careful observation of TEM images suggested that contrary to filament growth for K18 WT, which appeared to occur mainly through a vertical (end-to-end) growth mechanism (due to their generally long and slender morphology), K18 C291A/C322A/I260C had a lateral growth mechanism evidenced by regular lateral contacts between filaments, similar to those reported for amyloid $\beta$ with an $\mathrm{N}$ terminal cysteine. ${ }^{75}$ Quantitatively, 90\% (45/50) of fibrils formed by K18 C291A/C322A/I260C made lateral contacts with at least one other fibril. To the contrary, only $12 \%(5 / 41)$ lateral contacts were recorded for K18 WT. Figure $8 \mathrm{C}$ shows a typical TEM field of view for K18 WT filaments, demonstrating their long appearance without lateral contacts. Figure $8 \mathrm{D}$ shows an example of laterally interacting K18 C291A/C322A/I260C filament: wider but shorter than the K18 WT filament in Figure 8C. Our finding of vertical growth mechanism for K18 WT filaments is supported by a recent study that demonstrated this mechanism for FL tau-441. ${ }^{76}$ 
Aggregation of Triple Cysteine-Modified Tau K18 Is Characterized by Increased $\boldsymbol{\beta}$-Sheet Structures. It is wellknown that the aggregation of K18 WT results in secondary structure rearrangement toward ordered $\beta$-sheet conformers. ${ }^{53,59,77,78}$ We therefore investigated if K18 C291A/C322A/ I260C also had this property. K18 C291A/C322A/I260C monomers had negative $\mathrm{CD}$ spectra with the mean minimum peak at $199.00 \pm 0.55 \mathrm{~nm}$, similar to that of K18 WT at 200.70 $\pm 0.15 \mathrm{~nm}$ (Figure S5A,B), suggesting predominant random coil conformations $^{59,77,78}$ and indicating that the triple cysteine modification does not alter the unfolded structure of tau K18 monomers. Following heparin-induced aggregation without shaking, the K18 C291A/C322A/I260C spectra shifted toward longer wavelengths, with new mean peaks of $202.90 \pm 1.07 \mathrm{~nm}$ at day 4 and $206.90 \pm 0.03 \mathrm{~nm}$ at day 8 (Figure 9A and Table
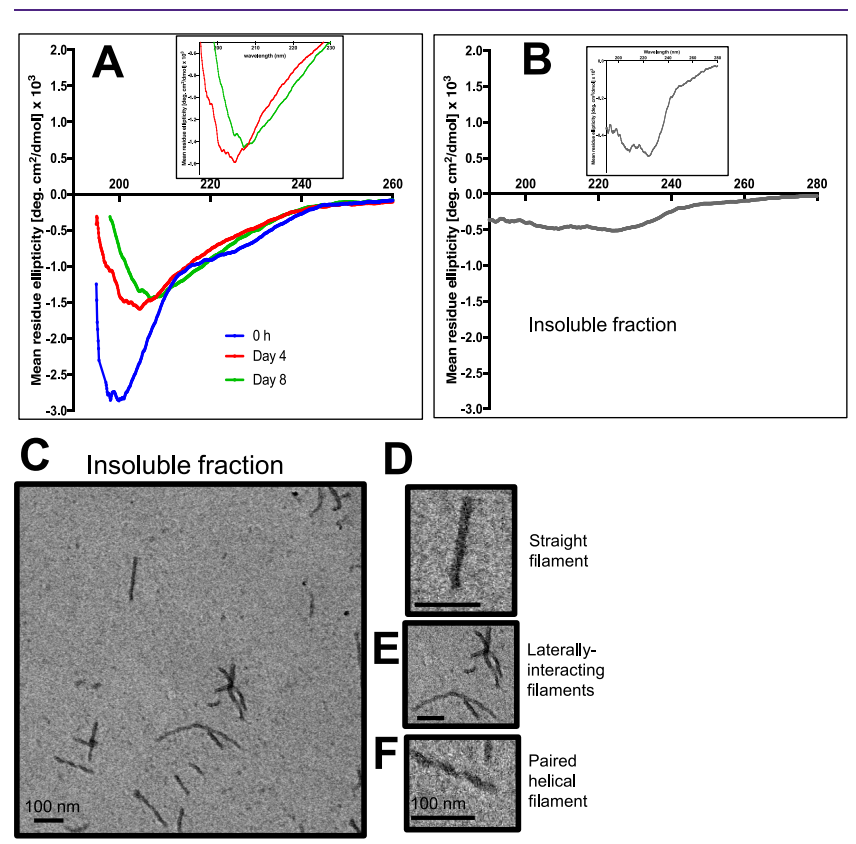

Figure 9. Aggregation of tau K18 C291A/C322A/I260C is characterized by progressive $\beta$-sheet enrichment. (A) $\mathrm{CD}$ analysis of time-dependent secondary structure changes in tau K18 C291A/ C322A/I260C during aggregation. Mean negative peak signals increased from $196.60 \pm 0.71 \mathrm{~nm}$ at day 0 to $202.90 \pm 1.07 \mathrm{~nm}$ and $206.90 \pm 0.03 \mathrm{~nm}$ at days 4 and 8 , respectively. The inset image highlights peak intensity transition from day 4 to day 8 . Each plot shows the mean of $n=3$ repeats with quantitative data given in Table S5. (B) Mean CD spectrum of secondary structure content of the insoluble fraction of aggregated tau K18 C291A/C322A/I260C showing peak intensity at $224.00 \pm 1.10 \mathrm{~nm}(n=2$, quantitative data provided in Table S6). The inset highlights the spectrum peak on a shorter vertical axis. (C) TEM characterization of the insoluble aggregate fraction from (B). Similar to data shown in Figures 8 and 9, K18 C291A/C322A/I260C formed a mix of PHFs and SFs. The images in (D), (E), and (F) are zoomed in regions from (C). Scale bars $=100 \mathrm{~nm}$.

S5). Insoluble aggregates (resuspended pellets) had negative spectra with a mean peak at $224.00 \pm 1.10 \mathrm{~nm}$ (Figure 9B and Table S6). The K18 C291A/C322A/I260C insoluble aggregate fraction consisted of abundant filaments (Figure 9C), including SFs (Figure 9D) and PHFs (Figure 9F) and laterally interacting filaments (Figure 9E) similar to those described in Figures 7 and 8. Together, $\beta$-sheet content in K18
C291A/C322A/I260C increased progressively with aggregation.

\section{CONCLUSION}

The preparation of recombinant forms of tau requires sequence-verified plasmids for overexpressing the protein in suitable expression hosts such as E. coli. ${ }^{59,79,80}$ The main aim of this study was to design, produce, and characterize a large library of plasmids to simplify the preparation of recombinant FL and truncated tau protein variants. A total of 44 sequenceverified plasmids were generated. These include plasmids for expressing WT and FTD tau (V337M, N279K, P301L, C291R, $\mathrm{S} 356 \mathrm{~T})$ and their respective cysteine-modified variants (C291A, C291A/C322A, C291A/C322A/I260C) on FL and K18 backgrounds. Moreover, we have established protocols that ensure high-level expression and also demonstrate the purification and initial biochemical characterization of a representative selection of FL and truncated tau genetic constructs.

In this study, we focused on FL tau-441 and K18, both of the $4 \mathrm{R}$ tau background. More than 110 MAPT mutations have been described, with more of such mutations occurring in $4 R$ than $3 \mathrm{R}$ tau isoforms. ${ }^{19} 4 \mathrm{R}$ tau has superior MT binding ability compared with $3 \mathrm{R} \mathrm{tau}{ }^{14}$ and is a common constituent of PHFs and NFTs isolated from the brains of individuals who died with many forms of neurodegenerative tauopathies. ${ }^{67,81-83}$ Moreover, truncation of $4 \mathrm{R}$ tau into fragments containing whole or part of the MTBR is a post-translational modification with probable biomarker importance. ${ }^{84,85} 4 \mathrm{R}$ tau therefore offers an opportunity to study the molecular origins of MT binding, aggregation, and filament formation, with direct relevance to the protein's physiological and pathophysiological functions.

Verified Plasmids and Protocols for Expressing and Purifying FL and Truncated Tau. Our comprehensive plasmid library is meant to help address common bottlenecks in plasmid sharing by providing ready-to-use genetic resources with accompanying protocols for generating FL and truncated tau variants. With the described protocols, the expression and purification process is achievable in 2 or 3 days for $6 x$ Histagged and 6xHis tag-free tau, respectively, far shorter than other published protocols. ${ }^{79,80}$ We expect that this first-of-akind open access genetic resource library and the accompanying methods will facilitate further studies. The expression and purification protocols described are representative for the FL and truncated tau forms created, as demonstrated above for the cysteine-modified constructs and previously for the N279K, V337M, and C291R variants. ${ }^{35,58,59,86}$

Impact of Triple Cysteine Modification on Tau K18 Aggregation and Filament Formation. Commonly used fluorescent protein tags such as green fluorescent protein can lead to unintended cellular effects, ${ }^{87,88}$ which in addition to their large molecular weights $(\sim 27 \mathrm{kDa})$ makes them unideal as reporter systems for internalization and release of exogenous tau. For this reason, the triple cysteine-modified forms of tau have become an increasingly popular set of tools for studying the aggregation, cellular internalization, release, and propagation of these proteins in real time because of the advantage of labeling a single cysteine residue located outside the MTBR with fluorescent compounds. ${ }^{49,53-57}$ In addition, we recently reported that specific labeling of triple cysteine-modified tau K18 with the maleimide derivatives $\mathrm{N}$-ethyl maleimide or Alexa Fluor C3-maleimide in cold conditions generates stable 
tau oligomers suitable for biochemical, cellular, and electrophysiological studies. ${ }^{35,58,89,90}$ However, it is unknown how the in vitro aggregation mechanism compares to that of K18 WT. We therefore took advantage of the resources and methods created to investigate potential effects of the triple cysteine modification on the aggregation paths and products of tau $\mathrm{K} 18$. Some earlier studies reported that $4 \mathrm{R}$ tau isoforms have decreased aggregation kinetics compared to $3 \mathrm{R}$ isoforms, which may be due to the two native cysteine residues in $4 R$ tau forming aggregation-incompetent intramolecular disulfide bonds (the so-called compact monomer conformation). ${ }^{29}$ However, others have argued that intramolecular disulfide bonds may not have significant effects on $4 \mathrm{R}$ tau aggregation and filament formation. ${ }^{49,52}$ Here, we sought to help address this unresolved question by studying if the number of cysteine residues influences tau K18 aggregation kinetics and filament structure. While some previous studies have compared $3 \mathrm{R}$ and $4 \mathrm{R}$ tau isoforms and constructs directly, ${ }^{29}$ the deletion of an entire repeat domain may have affected their experimental outcomes, for instance, given the crucial pro-aggregation role of the ${ }^{275} \mathrm{VQIINK}{ }^{280}$ hexapeptide motif in the second repeat domain which is absent in $3 \mathrm{R}$ tau. $^{31,91}$ Given that the K18 region is required for both disulfide-dependent and disulfideindependent tau aggregation, ${ }^{92}$ we studied the double-cysteine WT fragment and its C291A/C322A/I260C single-cysteine variant which differ in the number of cysteine residues. Although we cannot rule out potential effects of cysteine positional changes in this variant, the present study was focused on understanding disulfide bonding contributions to tau K18 aggregation based on the number of cysteine residues. This enabled detailed biochemical characterization of the in vitro aggregation properties of K18 C291A/C322A/I260C. Our findings indicate that tau $\mathrm{K} 18 \mathrm{WT}$, with two cysteine residues, aggregates into filaments more rapidly than its singlecysteine variant. This is in agreement with a previous study which demonstrated that the $3 \mathrm{R}$ FL tau (which has a single native cysteine) forms less abundant dimers and higher order oligomers compared to the $4 \mathrm{R}$ FL tau. ${ }^{92}$ An explanation for this result is that as $\mathrm{K} 18 \mathrm{C} 291 \mathrm{~A} / \mathrm{C} 322 \mathrm{~A} / \mathrm{I} 260 \mathrm{C}$ contains a single cysteine, it can only form disulfide-dependent dimers without disulfide-dependent higher-order aggregates compared to the WT which can aggregate into larger structures via the disulfide-dependent pathway ${ }^{27}$ (Figure 10). K18 WT therefore had a faster aggregation due to combined effects of disulfidedependent and -independent aggregation processes compared to K18 C291A/C322A/I260C with weaker disulfide-dependent activity.

An alternative interpretation for our results is that the ability of tau K18 to form filamentous aggregates is independent of whether a cysteine residue is located within the first, second, or third repeat domains. Previous studies on the contribution of disulfide bonds to $4 \mathrm{R}$ tau aggregation modified single or double cysteine residues to nonpolar amino acids like alanine. $^{26-28,51}$ Nonetheless, effects of introducing cysteine residues outside the second and third repeat domains have hitherto been unknown. We have shown here that restricting cysteine residues to the second and third repeat regions (for K18 WT) is not a requirement for Alzheimer-like filament formation in vitro. Rather, aggregation characterized by $\beta$ sheet-dependent filament assembly is attainable, although at a reduced rate, when a single cysteine residue is placed in the first repeat domain.
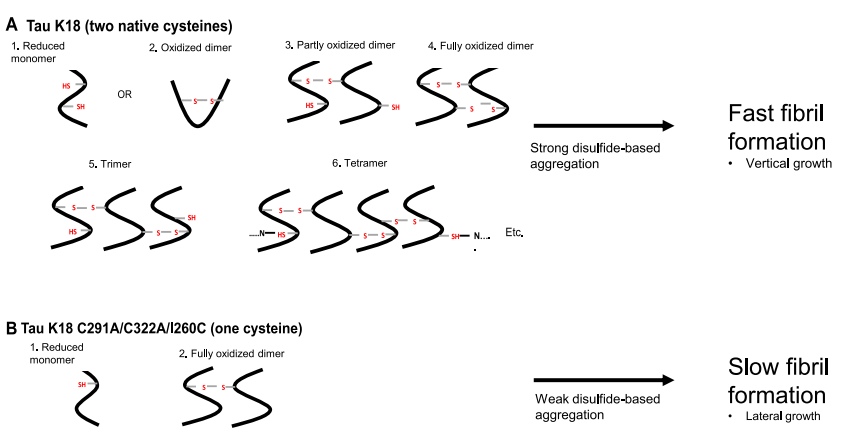

Figure 10. A proposed model of cysteine-dependent tau K18 aggregation in nonreducing conditions. (A) Monomeric tau K18 WT, with its two native cysteine residues, can undergo either intramolecular disulfide bonding to form "compact monomers" or intermolecular disulfide bonding to form aggregation-competent dimers. Partially oxidized dimers, but not fully oxidized dimers, can undergo further cysteine-dependent aggregation with reduced monomers to form trimers, tetramers, and correspond larger aggregates. (B) Tau K18 C291A/C322A/I260C contains a single cysteine residue and for that matter can only form an oxidized dimer, which cannot further aggregate via the cysteine-dependent pathway. Since both proteins have intact hexapeptide motifs that also modulate aggregation, it is proposed that the strong cysteine-dependent aggregation tendency of tau K18 explains its overall higher aggregation and filament-forming tendency compared to K18 C291A/C322A/I260C.

An important finding from this study is that a specific rearrangement of native cysteine residues resulting in a single cysteine outside the MTBR can drastically decrease the aggregation kinetics and delay filament assembly in tau K18. Similarly, modification of the C291 residue of K18 WT to serine inhibited the peptide's oligomerization competence. ${ }^{27}$ The strategy of modifying cysteine residues may therefore inform the design of effective approaches to inhibit $4 \mathrm{R}$ tau aggregation. Compared to the computer-guided random amino acid shuffling approach used to generate aggregationcompromised scrambled proteinopathic proteins, ${ }^{93,94}$ cysteine modification is a more reproducible strategy that uses a nonpharmacological approach to inhibit tau K18 aggregation, with its effectiveness independent of external agents. Given the physiological and pathophysiological importance of $4 \mathrm{R}$ tau, ${ }^{16,95,96}$ a viable approach to block its disease-driving aggregation will be useful for targeting most tauopathies.

The observed similarities and differences in filament morphology for K18 WT and K18 C291A/C322A/I260C suggest that the two protein variants have both shared and divergent conformational changes leading to filamentous aggregation. The mechanisms underlying these similar-yetdiverse properties are not currently understood. However, a few features stand out. K18 WT mostly formed long and thin filaments. Filament assembly by this peptide involved vertical association between two short filaments, as also reported in a different study. ${ }^{76}$ In contrast, K18 C291A/C322A/I260C filament elongation was dominated by lateral interactions of filaments. As demonstrated by TEM imaging (Figure 8D), coalescence of laterally associated filaments appeared responsible for the generally wide nature of the resulting mature filaments and may explain the frequently short appearance of K18 C291A/C322A/I260C filaments. Importantly, the lateral growth mechanism has been described for amyloid $\beta$ with an N-terminal cysteine, ${ }^{75}$ which highlights the importance of a single cysteine residue for this aggregation pathway. To our 
knowledge, however, this is the first description of this mechanism for a tau form. Notwithstanding their apparent distinct principal filament formation mechanisms, K18 WT and K18 C291A/C322A/I260C formed some filaments with the same structural phenotypes. This raises the possibility that filaments of the same structural phenotypes can be formed through apparent heterogeneous aggregation paths.

Based on our findings, we propose a new model of cysteinedependent tau aggregation (Figure 10). In nonreducing conditions, K18 WT can form either an intramolecular disulfide bond (compact monomer) or an intermolecular disulfide-based dimer as its least complex aggregate. Compact monomers do not appear to impede aggregation, potentially due to a low abundance or unfavorable formation in the presence of heparin. Dimers can associate with reduced monomers to form trimers and corresponding higher aggregates by disulfide linkages. Partially oxidized dimers/ trimers/tetramers/larger oligomers can also cointeract through disulfide bridges, therefore accelerating the rate of aggregation (Figure 10). For K18 C291A/C322A/I260C, compact monomer formation is not feasible, but a dimer is the only cysteine-dependent aggregate it can form (Figure 10). Since both constructs can theoretically undergo cysteine-independent aggregation to the same extent (each has intact aggregation-promoting hexapeptide motifs in the MTBR), we hypothesize that the stronger aggregation tendency of K18 WT is conferred by its higher potential of cysteine-dependent polymerization. It is currently unclear if these disulfide linkages have any association with the filament assembly mechanisms observed.

A limitation of this work is that even though the K18 fragment allows for accelerated aggregation, using this truncated construct inevitably simplifies the otherwise more complicated process for FL tau. Therefore, not all aspects of the functional characterization described here for tau K18 may apply to FL tau. Nonetheless, the described plasmids and biochemical protocols will allow complementary FL tau versions of the K18 constructs to be produced and characterized.

In conclusion, we expect that the tau plasmid library and the associated expression and purification protocols described herein will facilitate future studies into the structural and molecular determinants of the protein's MT binding in physiological conditions and aggregation in multiple diseases and aid the design of effective inhibition agents. Moreover, the new insights provided into the mechanistic basis of tau K18 filament polymorphisms should pave the way for further studies exploring this knowledge for therapeutic gains.

\section{METHODS}

Materials. Heparin (\#H3393), sodium phosphate dibasic (\#S9763), sodium phosphate monobasic dihydrate (\#4269), and thioflavin T (\#T3516) were obtained from Sigma-Aldrich (St. Louis, Missouri, USA). The QIAprep Spin Miniprep kit and the Q5 sitedirected mutagenesis kit (\#E0554S) were bought from QIAGEN GmbH (Hilden, Germany) and New England Biolabs (Ipswich, Massachusetts, USA), respectively. The Bicinchoninic acid assay kit (\#786-570) was obtained from G-Biosciences (St. Louis, Missouri, USA). The K9JA total tau antibody (\#A0024) was purchased from Dako (now Agilent), 77G7 (\#816701) and Tau12 (\#806501) were purchased from BioLegend (San Diego, California, USA), and fast flow resin with chelating sepharose (\#17-0575-01) was purchased from GE Healthcare (Buckinghamshire, UK). NuPAGE 4-12\% Novex Bis-Tris gels (\#NP0321), 4× LDS (lithium dodecyl sulfate) buffer (\#NP0007), 20× MES (2-(N-morpholino)ethanesulfonic acid) SDS running buffer (\#NP0002), and 10× sample reducing agent (\#NP0004) were purchased from ThermoFisher. HRP-labeled secondary antibody (\#W4021) was purchased from Promega. Isopropyl $\beta$-D-1-thiogalactopyranoside (IPTG; \#I6758) and DNase I (\#04716728001) were purchased from Sigma, while BugBuster (\#70921) and protease inhibitor cocktail (\#11836153001) were purchased from Merck. BL21(DE3)*pRosetta cells and TEV protease were kind gifts of Prof. David Roper and Dr. Deborah Brotherton, respectively, both at the University of Warwick, Coventry, UK.

Plasmid Cloning, Protein Expression, and Purification. Plasmid design, cloning, site-directed mutagenesis, transformation, and DNA sequencing have been described elsewhere. ${ }^{59}$ Amino acid changes made and primers used for site-directed mutagenesis have been listed in Tables S2 and S3. Plasmids meant for open-access sharing were prepared by transformation into BL21(DE3)*pRosetta cells and amplified by overnight growth in Luria-Bertani broth containing ampicillin $(100 \mu \mathrm{g} / \mathrm{mL})$ and chloramphenicol $(35 \mu \mathrm{g} /$ $\mathrm{mL}$ ), and the DNA was isolated using the QIAprep Spin Miniprep kit following the manufacturer's instructions. DNA concentration was determined on a NanoDrop NP60 spectrophotometer. Mutagenic changes were confirmed by aligning DNA sequencing chromatograms against the template plasmid sequences using Genome Compiler (Twist Bioscience, California, USA). Plasmid DNA aliquots are available for sharing, allowing their transformation into the host of choice. Details of plasmids can be found in Table S1.

Plasmids transformed into BL21(DE3)*pRosetta cells were expressed and first purified by IMAC as described. ${ }^{59}$ Briefly, an overnight culture of $10 \mathrm{~mL}$ of selective $\mathrm{LB}$ broth inoculated with single colonies of BL21(DE3)*pRosetta cells encoding the tau plasmid of interest was used to inoculate a $1 \mathrm{~L}$ fresh selective $\mathrm{LB}$ broth. The cultures were grown at $37{ }^{\circ} \mathrm{C}$ with $180 \mathrm{rpm}$ shaking until the $\mathrm{OD}_{600}$ reached 0.6-07. After inducing expression with $0.5 \mathrm{mM}$ IPTG, the cultures were returned to the $37{ }^{\circ} \mathrm{C}$ shaking incubator for a further $1 \mathrm{~h}$. The cell lysates were harvested by centrifuging the cultures at $9800 \times \mathrm{g}$ for $10 \mathrm{~min}$ at $4{ }^{\circ} \mathrm{C}$. The recovered pellets were resuspended in $50 \mathrm{mM}$ sodium phosphate buffer $\mathrm{pH} 7.5$ and frozen at $-20{ }^{\circ} \mathrm{C}$ until use. Immediately before purification, the lysates were heated at $70{ }^{\circ} \mathrm{C}$ in the presence of the following reagents added per $50 \mathrm{~mL}$ lysate: $5 \mathrm{~mL}$ of $1 \times$ BugBuster, $10 \mathrm{mg}$ DNase I, and 1 tablet protease inhibitor cocktail. The mixtures were kept at room temperature for $1 \mathrm{~h}$ and then sonicated (70\% power, $1 \mathrm{~min})$ in a Bandelin Sonopuls 2070 sonicator and centrifuged $(48000 \times \mathrm{g}, 30$ min, $\left.4{ }^{\circ} \mathrm{C}\right)$. The supernatant was decanted and filtered $(0.2 \mu \mathrm{m})$ before IMAC purification.

Chelating Sepharose resin packed into a Ni-NTA column (BioRad) was charged with $10 \mathrm{mM} \mathrm{NiCl} / \mathrm{CH}_{3} \mathrm{COONa} \mathrm{pH} 4.0$ buffer and equilibrated with two column volumes $(\mathrm{CVs})$ of buffer A $(50 \mathrm{mM}$ sodium phosphate buffer $\mathrm{pH} 7.0,500 \mathrm{mM} \mathrm{NaCl}, 10 \mathrm{mM}$ imidazole). After applying the crude extract (input), unbound protein was removed in a two-step washing protocol, first with two CVs of buffer $\mathrm{A}$ and then 2 CVs of buffer B ( $50 \mathrm{mM}$ sodium phosphate buffer $\mathrm{pH}$ 7.0, $500 \mathrm{mM} \mathrm{NaCl}, 25 \mathrm{mM}$ imidazole). His-tagged proteins were eluted with buffer $\mathrm{C}$ ( $50 \mathrm{mM}$ sodium phosphate buffer $\mathrm{pH} 7.0,500$ $\mathrm{mM} \mathrm{NaCl}, 500 \mathrm{mM}$ imidazole, $10 \mathrm{mM}$ dithiothreitol). Selected fractions, as analyzed by SDS-PAGE and Western blotting, were pooled and concentrated by ultrafiltration using 3 and $10 \mathrm{kDa}$ cutoff centrifugal filter devices (Amicon) for K18 and FL tau, respectively. All constructs were initially further purified by size exclusion chromatography (SEC) on a Superdex S200 10/300 GL column (\#17-5175-01, GE Healthcare) running on an Ethan LC system (GE Healthcare) with $1 \times \mathrm{PBS}$ pH 7.4 as the running buffer. The molecular weights of the fractions of interest were estimated using the elution volumes of the following size markers from Sigma (\#MWGF70-1KT): blue dextran (2000 $\mathrm{kDa}$; void volume), bovine serum albumin (66 $\mathrm{kDa})$, carbonic anhydrase $(29 \mathrm{kDa})$, cytochrome $\mathrm{C}(12.4 \mathrm{kDa})$, and aprotinin $(6.5 \mathrm{kDa})$. The optimized process showed that a SEC polishing step was not necessary for K18 (data not shown). For all constructs, the $6 \mathrm{x}-\mathrm{His}$ tag was removed by overnight dialysis against dialysis buffer ( $50 \mathrm{mM}$ Tris $\mathrm{HCl} \mathrm{pH} 7.5,100 \mathrm{mM} \mathrm{NaCl}$ ) in the 
presence of $25 \mu \mathrm{g} / \mathrm{mL}$ TEV protease. The cleavage products were separated by applying the dialyzed preparation to a charged resin equilibrated as previously and collecting the cleaved tau as the flowthrough.

Western Blot. Equal volumes of SEC fractions were diluted in the LDS sample buffer and the sample reducing agent and run in $4-12 \%$ Bis-Tris protein gels in MES SDS running buffer (gel and buffers were purchased from ThermoFisher Scientific; see the Materials section above). The gels were subsequently equilibrated in transfer buffer (20\% methanol) for $25 \mathrm{~min}$. Proteins were transferred to a polyvinylidene fluoride membrane which had been previously activated in pure methanol for $30 \mathrm{~s}$ and soaked in the transfer buffer for another $25 \mathrm{~min}$. Transfer was performed in a semidry blot instrument at $0.8 \mathrm{~mA} / \mathrm{cm}^{2}$ for $60 \mathrm{~min}$ at room temperature. The membrane was then blocked for $1 \mathrm{~h}$ at room temperature using PBST-BSA (PBS with 0.05\% Tween 20 and 1\% BSA) with gentle shaking. Primary antibody incubation was performed overnight at 4 ${ }^{\circ} \mathrm{C}$ at a ratio of $1 \mu \mathrm{g}$ of antibody per $1 \mathrm{~mL}$ of PBST-BSA. Afterward, the membrane was washed three times with PBST (PBS with $0.05 \%$ Tween 20), with each wash lasting $10 \mathrm{~min}$ with light shaking. Secondary antibody was incubated at a ratio of $1 \mu \mathrm{L}$ per $10 \mathrm{~mL}$ of PBST-BSA for $1 \mathrm{~h}$ at room temperature with gentle shaking. Signal detection was achieved by adding Amersham electrochemiluminiscent detection reagents (GE Healthcare, Buckinghamshire, UK) and imaging with the LAS 3000 camera system (Fujifilm). Western blot characterization of the initial IMAC-based purification using the MTBR-binding K9JA antibody followed a previously published protocol. ${ }^{59}$

ThT Fluorescence Assay. Ten microliters each of heparin (MW 6000 ) and ThT (to yield $25 \mu \mathrm{M}$ and $20 \mu \mathrm{M}$ final concentrations, respectively) was added to $80 \mu \mathrm{L}$ of tau $\mathrm{K} 18 \quad(9 \mu \mathrm{M}$ final concentration) per well of a 96-well plate (Greiner Bio-One \#655077). Aggregation was monitored for $15 \mathrm{~h}$ by measuring ThT fluorescence at 440 and $510 \mathrm{~nm}$ in a CLARIOstar microplate reader (BMG Lab Tech, Ortenberg, Germany). Sensitivity was set to $20 \%$ to prevent signal saturation. Reading using the top optic setting was obtained every $5 \mathrm{~min}$ after $200 \mathrm{rpm}$ shaking for $30 \mathrm{~s}$. The ThT signal at each time point was normalized to that of buffer only by subtracting the absorbance value of the blank at each identical time point from those of samples. Unless otherwise stated, the buffer used in all assays was $10 \mathrm{mM}$ sterile-filtered sodium phosphate buffer pH 7.4.

Filament Assembly and Characterization by Negative-Stain TEM. Freshly purified samples $(30 \mu \mathrm{M})$ were incubated with $63 \mu \mathrm{M}$ heparin for 5 or 16 days at $37^{\circ} \mathrm{C}$ without shaking. Samples were then diluted 1:2 with $10 \mathrm{mM}$ sodium phosphate buffer $\mathrm{pH} 7.4$ and analyzed by TEM as described. ${ }^{59}$ The Image freeware was used for filament length and width analysis as follows: after setting the scale, the freehand line tool was used to trace filament architecture, and the length was then measured. The straight line tool was used for width measurements. For filaments of apparently even width, measurements were taken once from random positions. For those coalescing from two filaments or of otherwise uneven widths, measurements were taken from representative spots as described in Figure 9D. We followed the terminology used by $\mathrm{Xu}$ et al. $(2010)^{74}$ in characterizing fibril structures.

CD Spectroscopy. Tau K18 samples $(200 \mu \mathrm{L})$ diluted in $10 \mathrm{mM}$ sodium phosphate buffer $\mathrm{pH} 7.4$ were transferred to a $1 \mathrm{~mm}$ pathlength cuvette and analyzed on a Jasco J-815 CD spectropolarimeter (Jasco Inc., Maryland, USA) with $1 \mathrm{~s}$ response time, $0.1 \mathrm{~nm}$ data pitch, and $100 \mathrm{~nm} / \mathrm{min}$ scan speed. Averaged spectral accumulations were collected at 190 to $280 \mathrm{~nm}$ wavelengths, with the high-tension voltage maintained at $\leq 550 \mathrm{~V}$.

$\mathrm{CD}$ analysis of monomers involved $15 \mu \mathrm{M} \mathrm{K18} \mathrm{WT}$ and K18 C291A/C322A/I260C. For time-dependent CD traces, data were taken on $20 \mu \mathrm{M}$ monomers on day 0 after which an $83 \mu \mathrm{M}$ final concentration of heparin was added, and the mixture was incubated at $37{ }^{\circ} \mathrm{C}$ without shaking. $\mathrm{CD}$ measurements were repeated on days 4 and 8 . In sedimentation assays, filament samples were ultracentrifuged at $100,000 \times \mathrm{g}$ for $1 \mathrm{~h}$ at $4{ }^{\circ} \mathrm{C}$, the supernatant was removed, and the pellet was resuspended in $200 \mu \mathrm{L}$ of $10 \mathrm{mM}$ sodium phosphate buffer $\mathrm{pH} 7.4$ for CD analysis.

Statistics. Data are expressed as mean \pm standard error of the mean (SEM) from $n=3$ experiments, unless otherwise stated. Data analysis and graph preparation were performed using Prism 7 (GraphPad Inc., California, USA). Statistical comparison of filament features was performed using the Mann-Whitney test at the 95\% confidence interval, as the data did not follow Gaussian distribution.

\section{ASSOCIATED CONTENT}

Supporting Information

The Supporting Information is available free of charge at https://pubs.acs.org/doi/10.1021/acschemneuro.0c00469.

Description of library of tau plasmids generated, primers used to generate plasmids for expressing and sequencing tau protein variants, Western blot and SEC characterization of expression and purification of tau variants, and CD spectroscopy characterization of soluble and insoluble tau aggregates (PDF)

\section{AUTHOR INFORMATION}

\section{Corresponding Author}

Thomas K. Karikari - School of Life Sciences and Midlands Integrative Biosciences Training Partnership, University of Warwick, Coventry CV4 7AL, U.K.; Department of Psychiatry and Neurochemistry, Institute of Neuroscience and Physiology, The Sahlgrenska Academy, University of Gothenburg, Gothenburg SE 43180, Sweden; 이이. org/0000-00031422-4358; Email: Thomas.Karikari@gu.se

\section{Authors}

Sophie Keeling - School of Life Sciences, University of Warwick, Coventry CV4 7AL, U.K.

Emily Hill - School of Life Sciences, University of Warwick, Coventry CV4 7AL, U.K.

Juan Lantero Rodríguez - Department of Psychiatry and Neurochemistry, Institute of Neuroscience and Physiology, The Sahlgrenska Academy, University of Gothenburg, Gothenburg SE 43180, Sweden

David A. Nagel - School of Life and Health Sciences, Aston University, Birmingham B4 7ET, U.K.

Bruno Becker - Department of Psychiatry and Neurochemistry, Institute of Neuroscience and Physiology, The Sahlgrenska Academy, University of Gothenburg, Gothenburg SE 43180, Sweden; Clinical Neurochemistry Laboratory, Sahlgrenska University Hospital, Mölndal 431 80, Sweden

Kina Höglund - Department of Psychiatry and Neurochemistry, Institute of Neuroscience and Physiology, The Sahlgrenska Academy, University of Gothenburg, Gothenburg SE 43180, Sweden; Clinical Neurochemistry Laboratory, Sahlgrenska University Hospital, Mölndal 431 80, Sweden

Henrik Zetterberg - Department of Psychiatry and Neurochemistry, Institute of Neuroscience and Physiology, The Sahlgrenska Academy, University of Gothenburg, Gothenburg SE 43180, Sweden; Clinical Neurochemistry Laboratory, Sahlgrenska University Hospital, Mölndal 431 80, Sweden; Department of Neurodegenerative Disease, UCL Institute of Neurology, London WC1E 6BT, U.K.; UK Dementia Research Institute at UCL, London WC1E 6BT, U.K.

Kaj Blennow - Department of Psychiatry and Neurochemistry, Institute of Neuroscience and Physiology, The Sahlgrenska Academy, University of Gothenburg, Gothenburg SE 43180, 
Sweden

Eric J. Hill - School of Life and Health Sciences, Aston University, Birmingham B4 7ET, U.K.

Kevin G. Moffat - School of Life Sciences, University of Warwick, Coventry CV4 7AL, U.K.

Complete contact information is available at: https://pubs.acs.org/10.1021/acschemneuro.0c00469

\section{Author Contributions}

T.K.K., S.K., E.H., J.L.R., D.A.N., and B.B. performed the experiments and analyzed data. K.H., H.Z., K.B., E.J.H., and K.G.M. provided supervision, intellectual contributions, and contributed reagents. T.K.K. wrote the first manuscript draft which was edited by D.A.N., H.Z., K.B., E.J.H., and K.G.M. All authors read, substantially contributed to, and approved the manuscript for submission.

\section{Funding}

This study was partly funded by the UK NC3Rs through the CRACK IT: Untangle grant (\#NC/C013101/1). T.K.K. was supported by the University of Warwick Chancellor's Scholarship, the Biotechnology and Biological Sciences Research Council (\#BB/J014532/1) through the Midlands Integrative Biosciences Training Partnership, the BrightFocus Foundation (\#A2020812F), the Swedish Brain Foundation (Hjärnfonden; \#FO2020-0240), the Swedish Alzheimer Foundation (\#AF930627), the Swedish Dementia Foundation (Demensförbundet), the Agneta Prytz-Folkes \& Gösta Folkes Foundation (\#2020-00124), Gamla Tjänarinnor Foundation, the Aina (Ann) Wallströms and Mary-Ann Sjöbloms Foundation, the Gun \& Bertil Stohnes Foundation, and the Anna Lisa and Brother Björnsson's Foundation. Open access funding was generously provided by the RCUK block grant at the University of Warwick. The funders had no role in study design, data collection, and analysis, decision to publish, or preparation of the manuscript.

\section{Notes}

The authors declare no competing financial interest.

Supporting research data regarding 44 sequence-verified plasmids for this article may be requested from the corresponding author by completing the following form: ht t p s : / / d ocs.google.com/forms/d/e / 1FAIpQLSfcr1 z 3 LF 0v8EVxvPoyf6tKaWIdktX_qmP1LPySEBFl99OMw/viewform?usp=pp_url.

\section{ACKNOWLEDGMENTS}

We thank Prof. David Roper and Dr. Deborah Brotherton, both of the University of Warwick, for the gift of BL21(DE3)*pRosetta cells and TEV protease, respectively. The pCMV-tau plasmids were kind gifts of Dr. Calum Sutherland of the University of Dundee, Dundee, UK.

\section{ABBREVIATIONS}

$\mathrm{AD}$, Alzheimer's disease; AFM, atomic force microscopy; $\mathrm{CV}$, column volumes; FL, full length; FTD, frontotemporal dementia; IMAC, immobilized metal affinity purification; K18, four-repeat tau fragment; MRE, mean residue ellipticity; IPTG, isopropyl $\beta$-D-1-thiogalactopyranoside; MT, microtubule; MTBR, MT-binding repeat; NFT, neurofibrillary tangle; PCR, polymerase chain reaction; PHF, paired helical filament; RFU, relative fluorescent units; SF, straight filament; SEC, size exclusion chromatography; SEM, standard error of the mean; TEM, transmission electron microscopy; ThT, thioflavin T; WT, wild type; WB, Western blot(ting); 4R, fourrepeat; $3 R$, three-repeat

\section{REFERENCES}

(1) Andreadis, A., Brown, W. M., and Kosik, K. S. (1992) Structure and Novel Exons of the Human Tau Gene. Biochemistry 31 (43), 10626-10633.

(2) Neve, R. L., Harris, P., Kosik, K. S., Kurnit, D. M., and Donlon, T. A. (1986) Identification of CDNA Clones for the Human Microtubule-Associated Protein Tau and Chromosomal Localization of the Genes for Tau and Microtubule-Associated Protein 2. Mol. Brain Res. 1 (3), 271-280.

(3) Goedert, M., Spillantini, M. G., Potier, M. C., Ulrich, J., and Crowther, R. A. (1989) Cloning and Sequencing of the CDNA Encoding an Isoform of Microtubule-Associated Protein Tau Containing Four Tandem Repeats: Differential Expression of Tau Protein MRNAs in Human Brain. EMBO J. 8 (2), 393-399.

(4) Goedert, M., Wischik, C. M., Crowther, R. A., Walker, J. E., and Klug, A. (1988) Cloning and Sequencing of the CDNA Encoding a Core Protein of the Paired Helical Filament of Alzheimer Disease: Identification as the Microtubule-Associated Protein Tau. Proc. Natl. Acad. Sci. U. S. A. 85 (11), 4051-4055.

(5) Andreadis, A. (2005) Tau Gene Alternative Splicing: Expression Patterns, Regulation and Modulation of Function in Normal Brain and Neurodegenerative Diseases. Biochim. Biophys. Acta, Mol. Basis Dis. 1739 (2), 91-103.

(6) Goedert, M., Spillantini, M. G., Jakes, R., Rutherford, D., and Crowther, R. A. (1989) Multiple Isoforms of Human MicrotubuleAssociated Protein Tau: Sequences and Localization in Neurofibrillary Tangles of Alzheimer's Disease. Neuron 3 (4), 519-526.

(7) Buée, L., and Delacourte, A. (1999) Comparative Biochemistry of Tau in Progressive Supranuclear Palsy, Corticobasal Degeneration, FTDP-17 and Pick's Disease. Brain Pathol. 9 (4), 681-693.

(8) Goedert, M., Spillantini, M. G., Cairns, N. J., and Crowther, R. A. (1992) Tau Proteins of Alzheimer Paired Helical Filaments: Abnormal Phosphorylation of All Six Brain Isoforms. Neuron 8 (1), $159-168$.

(9) Woerman, A. L., Aoyagi, A., Patel, S., Kazmi, S. A., Lobach, I., Grinberg, L. T., McKee, A. C., Seeley, W. W., Olson, S. H., and Prusiner, S. B. (2016) Tau Prions from Alzheimer's Disease and Chronic Traumatic Encephalopathy Patients Propagate in Cultured Cells. Proc. Natl. Acad. Sci. U. S. A. 113 (50), E8187-E8196.

(10) Falcon, B., Zivanov, J., Zhang, W., Murzin, A. G., Garringer, H. J., Vidal, R., Crowther, R. A., Newell, K. L., Ghetti, B., Goedert, M., and Scheres, S. H. W. (2019) Novel Tau Filament Fold in Chronic Traumatic Encephalopathy Encloses Hydrophobic Molecules. Nature 568 (7752), 420-423.

(11) Zhang, W., Tarutani, A., Newell, K. L., Murzin, A. G., Matsubara, T., Falcon, B., Vidal, R., Garringer, H. J., Shi, Y., Ikeuchi, T., Murayama, S., Ghetti, B., Hasegawa, M., Goedert, M., and Scheres, S. H. W. (2020) Novel Tau Filament Fold in Corticobasal Degeneration. Nature 580, 283-287.

(12) Liu, F., and Gong, C.-X. (2008) Tau Exon 10 Alternative Splicing and Tauopathies. Mol. Neurodegener. 3, 8.

(13) Brandt, R., and Lee, G. (1993) Functional Organization of Microtubule-Associated Protein Tau. Identification of Regions Which Affect Microtubule Growth, Nucleation, and Bundle Formation in Vitro. J. Biol. Chem. 268 (5), 3414-3419.

(14) Panda, D., Samuel, J. C., Massie, M., Feinstein, S. C., and Wilson, L. (2003) Differential Regulation of Microtubule Dynamics by Three- and Four-Repeat Tau: Implications for the Onset of Neurodegenerative Disease. Proc. Natl. Acad. Sci. U. S. A. 100 (16), 9548-9553.

(15) Kadavath, H., Hofele, R. V., Biernat, J., Kumar, S., Tepper, K., Urlaub, H., Mandelkow, E., and Zweckstetter, M. (2015) Tau Stabilizes Microtubules by Binding at the Interface between Tubulin Heterodimers. Proc. Natl. Acad. Sci. U. S. A. 112 (24), 7501-7506.

(16) Ghetti, B., Oblak, A. L., Boeve, B. F., Johnson, K. A., Dickerson, B. C., and Goedert, M. (2015) Frontotemporal Dementia Caused by 
Microtubule-Associated Protein Tau Gene (MAPT) Mutations: A Chameleon for Neuropathology and Neuroimaging. Neuropathol. Appl. Neurobiol. 41 (1), 24-46.

(17) Ingram, E. M., and Spillantini, M. G. (2002) Tau Gene Mutations: Dissecting the Pathogenesis of FTDP-17. Trends Mol. Med. 8 (12), 555-562.

(18) Hutton, M., Lendon, C. L., Rizzu, P., Baker, M., Froelich, S., Houlden, H., Pickering-Brown, S., Chakraverty, S., Isaacs, A., Grover, A., Hackett, J., Adamson, J., Lincoln, S., Dickson, D., Davies, P., Petersen, R. C., Stevens, M., de Graaff, E., Wauters, E., van Baren, J., Hillebrand, M., Joosse, M., Kwon, J. M., Nowotny, P., Che, L. K., Norton, J., Morris, J. C., Reed, L. A., Trojanowski, J., Basun, H., Lannfelt, L., Neystat, M., Fahn, S., Dark, F., Tannenberg, T., Dodd, P. R., Hayward, N., Kwok, J. B., Schofield, P. R., Andreadis, A., Snowden, J., Craufurd, D., Neary, D., Owen, F., Oostra, B. A., Hardy, J., Goate, A., van Swieten, J., Mann, D., Lynch, T., and Heutink, P. (1998) Association of Missense and 5'-Splice-Site Mutations in Tau with the Inherited Dementia FTDP-17. Nature 393 (6686), 702-705.

(19) Alzforum. MAPT Mutations. https://www.alzforum.org/ mutations $/$ search?genes $\% 5 \mathrm{~B} \% 5 \mathrm{D}=492 \&$ diseases=\&keywords-entry= \&keywords=\#results (accessed 2020-08-19).

(20) Elbaum-Garfinkle, S., Cobb, G., Compton, J. T., Li, X.-H., and Rhoades, E. (2014) Tau Mutants Bind Tubulin Heterodimers with Enhanced Affinity. Proc. Natl. Acad. Sci. U. S. A. 111 (17), 63116316.

(21) Combs, B., and Gamblin, T. C. (2012) FTDP-17 Tau Mutations Induce Distinct Effects on Aggregation and Microtubule Interactions. Biochemistry 51 (43), 8597-8607.

(22) Barghorn, S., Zheng-Fischhöfer, Q., Ackmann, M., Biernat, J., von Bergen, M., Mandelkow, E.-M., and Mandelkow, E. (2000) Structure, Microtubule Interactions, and Paired Helical Filament Aggregation by Tau Mutants of Frontotemporal Dementias†. Biochemistry 39 (38), 11714-11721.

(23) Li, B., Chohan, M. O., Grundke-Iqbal, I., and Iqbal, K. (2007) Disruption of Microtubule Network by Alzheimer Abnormally Hyperphosphorylated Tau. Acta Neuropathol. 113 (5), 501-511.

(24) Alonso, A. d. C., Zaidi, T., Novak, M., Grundke-Iqbal, I., and Iqbal, K. (2001) Hyperphosphorylation Induces Self-Assembly of $\tau$ into Tangles of Paired Helical Filaments/Straight Filaments. Proc. Natl. Acad. Sci. U. S. A. 98 (12), 6923-6928.

(25) Arriagada, P. V., Growdon, J. H., Hedley-Whyte, E. T., and Hyman, B. T. (1992) Neurofibrillary Tangles but Not Senile Plaques Parallel Duration and Severity of Alzheimer's Disease. Neurology 42 (3), 631-639.

(26) Bhattacharya, K., Rank, K. B., Evans, D. B., and Sharma, S. K. (2001) Role of Cysteine-291 and Cysteine-322 in the Polymerization of Human Tau into Alzheimer-like Filaments. Biochem. Biophys. Res. Commun. 285 (1), 20-26.

(27) Kim, D., Lim, S., Haque, Md. M., Ryoo, N., Hong, H. S., Rhim, H., Lee, D.-E., Chang, Y.-T., Lee, J.-S., Cheong, E., Kim, D. J., and Kim, Y. K. (2015) Identification of Disulfide Cross-Linked Tau Dimer Responsible for Tau Propagation. Sci. Rep. 5, 15231.

(28) Schweers, O., Mandelkow, E. M., Biernat, J., and Mandelkow, E. (1995) Oxidation of Cysteine-322 in the Repeat Domain of Microtubule-Associated Protein Tau Controls the in Vitro Assembly of Paired Helical Filaments. Proc. Natl. Acad. Sci. U. S. A. 92 (18), $8463-8467$.

(29) Barghorn, S., and Mandelkow, E. (2002) Toward a Unified Scheme for the Aggregation of Tau into Alzheimer Paired Helical Filaments $\dagger$. Biochemistry 41 (50), 14885-14896.

(30) Zhong, Q., Congdon, E. E., Nagaraja, H. N., and Kuret, J. (2012) Tau Isoform Composition Influences Rate and Extent of Filament Formation. J. Biol. Chem. 287 (24), 20711-20719.

(31) Li, W., and Lee, V. M.-Y. (2006) Characterization of Two VQIXXK Motifs for Tau Fibrillization in Vitro. Biochemistry 45 (51), $15692-15701$.

(32) Lathuilière, A., Valdés, P., Papin, S., Cacquevel, M., Maclachlan, C., Knott, G. W., Muhs, A., Paganetti, P., and Schneider, B. L. (2017)
Motifs in the Tau Protein That Control Binding to Microtubules and Aggregation Determine Pathological Effects. Sci. Rep. 7 (1), 13556.

(33) von Bergen, M., Friedhoff, P., Biernat, J., Heberle, J., Mandelkow, E.-M., and Mandelkow, E. (2000) Assembly of $\tau$ Protein into Alzheimer Paired Helical Filaments Depends on a Local Sequence Motif (306VQIVYK311) Forming $\beta$ Structure. Proc. Natl. Acad. Sci. U. S. A. 97 (10), 5129-5134.

(34) Xiang, S., Kulminskaya, N., Habenstein, B., Biernat, J., Tepper, K., Paulat, M., Griesinger, C., Becker, S., Lange, A., Mandelkow, E., and Linser, R. (2017) A Two-Component Adhesive Tau Fibrils Arise from a Combination of a Well-Defined Motif and Conformationally Flexible Interactions. J. Am. Chem. Soc. 139 (7), 2639-2646.

(35) Karikari, T. K., Nagel, D. A., Grainger, A., Clarke-Bland, C., Crowe, J., Hill, E. J., and Moffat, K. G. (2019) Distinct Conformations, Aggregation and Cellular Internalization of Different Tau Strains. Front. Cell. Neurosci. 13, 296.

(36) Strang, K. H., Croft, C. L., Sorrentino, Z. A., Chakrabarty, P., Golde, T. E., and Giasson, B. I. (2018) Distinct Differences in Prionlike Seeding and Aggregation between Tau Protein Variants Provide Mechanistic Insights into Tauopathies. J. Biol. Chem. 293, 24082421.

(37) Clavaguera, F., Akatsu, H., Fraser, G., Crowther, R. A., Frank, S., Hench, J., Probst, A., Winkler, D. T., Reichwald, J., Staufenbiel, M., Ghetti, B., Goedert, M., and Tolnay, M. (2013) Brain Homogenates from Human Tauopathies Induce Tau Inclusions in Mouse Brain. Proc. Natl. Acad. Sci. U. S. A. 110 (23), 9535-9540.

(38) Boluda, S., Iba, M., Zhang, B., Raible, K. M., Lee, V. M.-Y., and Trojanowski, J. Q. (2015) Differential Induction and Spread of Tau Pathology in Young PS19 Tau Transgenic Mice Following Intracerebral Injections of Pathological Tau from Alzheimer's Disease or Corticobasal Degeneration Brains. Acta Neuropathol. 129 (2), 221237.

(39) Karch, C. M., Jeng, A. T., and Goate, A. M. (2012) Extracellular Tau Levels Are Influenced by Variability in Tau That Is Associated with Tauopathies. J. Biol. Chem. 287 (51), 42751-42762.

(40) Šmić, G., Babić Leko, M., Wray, S., Harrington, C., Delalle, I., Jovanov-Milošević, N., Bažadona, D., Buée, L., de Silva, R., Di Giovanni, G., Wischik, C., and Hof, P. R. (2016) Tau Protein Hyperphosphorylation and Aggregation in Alzheimer's Disease and Other Tauopathies, and Possible Neuroprotective Strategies. Biomolecules 6 (1), 6.

(41) Hong, M., Zhukareva, V., Vogelsberg-Ragaglia, V., Wszolek, Z., Reed, L., Miller, B. I., Geschwind, D. H., Bird, T. D., McKeel, D., Goate, A., Morris, J. C., Wilhelmsen, K. C., Schellenberg, G. D., Trojanowski, J. Q., and Lee, V. M.-Y. (1998) Mutation-Specific Functional Impairments in Distinct Tau Isoforms of Hereditary FTDP-17. Science 282 (5395), 1914-1917.

(42) Lasagna-Reeves, C. A., Castillo-Carranza, D. L., Sengupta, U., Guerrero-Munoz, M. J., Kiritoshi, T., Neugebauer, V., Jackson, G. R., and Kayed, R. (2012) Alzheimer Brain-Derived Tau Oligomers Propagate Pathology from Endogenous Tau. Sci. Rep. 2, 700.

(43) Lasagna-Reeves, C. A., Castillo-Carranza, D. L., Sengupta, U., Clos, A. L., Jackson, G. R., and Kayed, R. (2011) Tau Oligomers Impair Memory and Induce Synaptic and Mitochondrial Dysfunction in Wild-Type Mice. Mol. Neurodegener. 6, 39.

(44) Guo, J. L., and Lee, V. M.-Y. (2011) Seeding of Normal Tau by Pathological Tau Conformers Drives Pathogenesis of Alzheimer-like Tangles. J. Biol. Chem. 286 (17), 15317-15331.

(45) Guo, J. L., Narasimhan, S., Changolkar, L., He, Z., Stieber, A., Zhang, B., Gathagan, R. J., Iba, M., McBride, J. D., Trojanowski, J. Q., and Lee, V. M. Y. (2016) Unique Pathological Tau Conformers from Alzheimer's Brains Transmit Tau Pathology in Nontransgenic Mice. J. Exp. Med. 213 (12), 2635-2654.

(46) Kar, S., Fan, J., Smith, M. J., Goedert, M., and Amos, L. A. (2003) Repeat Motifs of Tau Bind to the Insides of Microtubules in the Absence of Taxol. EMBO J. 22 (1), 70-77.

(47) Landino, L. M., Skreslet, T. E., and Alston, J. A. (2004) Cysteine Oxidation of Tau and Microtubule-Associated Protein-2 by Peroxynitrite MODULATION OF MICROTUBULE ASSEMBLY 
KINETICS BY THE THIOREDOXIN REDUCTASE SYSTEM. J. Biol. Chem. 279 (33), 35101-35105.

(48) Al-Hilaly, Y. K., Pollack, S. J., Vadukul, D., Citossi, F., Rickard, J. E., Simpson, M., Storey, J. M. D., Harrington, C. R., Wischik, C. M., and Serpell, L. C. (2017) Alzheimer's Disease-like Paired Helical Filament Assembly from Truncated Tau Protein Is Independent of Disulphide Cross-Linking. J. Mol. Biol. 429 (23), 3650-3665.

(49) Furukawa, Y., Kaneko, K., and Nukina, N. (2011) Tau Protein Assembles into Isoform- and Disulfide-Dependent Polymorphic Fibrils with Distinct Structural Properties. J. Biol. Chem. 286 (31), 27236-27246.

(50) Grüning, C. S. R., Mirecka, E. A., Klein, A. N., Mandelkow, E., Willbold, D., Marino, S. F., Stoldt, M., and Hoyer, W. (2014) Alternative Conformations of the Tau Repeat Domain in Complex with an Engineered Binding Protein. J. Biol. Chem. 289 (33), 2320923218.

(51) Huvent, I., Kamah, A., Cantrelle, F.-X., Barois, N., Slomianny, C., Smet-Nocca, C., Landrieu, I., and Lippens, G. A. (2014) Functional Fragment of Tau Forms Fibers without the Need for an Intermolecular Cysteine Bridge. Biochem. Biophys. Res. Commun. 445 (2), 299-303.

(52) Walker, S., Ullman, O., and Stultz, C. M. (2012) Using Intramolecular Disulfide Bonds in Tau Protein to Deduce Structural Features of Aggregation-Resistant Conformations. J. Biol. Chem. 287 (12), 9591-9600.

(53) Kumar, S., Tepper, K., Kaniyappan, S., Biernat, J., Wegmann, S., Mandelkow, E.-M., Müller, D. J., and Mandelkow, E. (2014) Stages and Conformations of the Tau Repeat Domain during Aggregation and Its Effect on Neuronal Toxicity. J. Biol. Chem. 289 (29), 2031820332.

(54) Michel, C. H., Kumar, S., Pinotsi, D., Tunnacliffe, A., GeorgeHyslop, P. S., Mandelkow, E., Mandelkow, E.-M., Kaminski, C. F., and Schierle, G. S. K. (2014) Extracellular Monomeric Tau Protein Is Sufficient to Initiate the Spread of Tau Protein Pathology. J. Biol. Chem. 289 (2), 956-967.

(55) Manger, L. H., Foote, A. K., Wood, S. L., Holden, M. R., Heylman, K. D., Margittai, M., and Goldsmith, R. H. (2017) Revealing Conformational Variants of Solution-Phase Intrinsically Disordered Tau Protein at the Single-Molecule Level. Angew. Chem., Int. Ed. 56 (49), 15584-15588.

(56) Margittai, M., and Langen, R. (2004) Template-Assisted Filament Growth by Parallel Stacking of Tau. Proc. Natl. Acad. Sci. U. S. A. 101 (28), 10278-10283.

(57) Shammas, S. L., Garcia, G. A., Kumar, S., Kjaergaard, M., Horrocks, M. H., Shivji, N., Mandelkow, E., Knowles, T. P. J., Mandelkow, E., and Klenerman, D. A. (2015) Mechanistic Model of Tau Amyloid Aggregation Based on Direct Observation of Oligomers. Nat. Commun. 6, 7025.

(58) Karikari, T. K., Nagel, D. A., Grainger, A., Clarke-Bland, C., Hill, E. J., and Moffat, K. G. (2019) Preparation of Stable Tau Oligomers for Cellular and Biochemical Studies. Anal. Biochem. 566, 67-74.

(59) Karikari, T. K., Turner, A., Stass, R., Lee, L. C. Y., Wilson, B., Nagel, D. A., Hill, E. J., and Moffat, K. G. (2017) Expression and Purification of Tau Protein and Its Frontotemporal Dementia Variants Using a Cleavable Histidine Tag. Protein Expression Purif. 130, 44-54.

(60) Barghorn, S., Zheng-Fischhöfer, Q., Ackmann, M., Biernat, J., von Bergen, M., Mandelkow, E.-M., and Mandelkow, E. (2000) Structure, Microtubule Interactions, and Paired Helical Filament Aggregation by Tau Mutants of Frontotemporal Dementias $\dagger$. Biochemistry 39 (38), 11714-11721.

(61) Hoover, B. R., Reed, M. N., Su, J., Penrod, R. D., Kotilinek, L. A., Grant, M. K., Pitstick, R., Carlson, G. A., Lanier, L. M., Yuan, L.-L., Ashe, K. H., and Liao, D. (2010) Tau Mislocalization to Dendritic Spines Mediates Synaptic Dysfunction Independently of Neurodegeneration. Neuron 68 (6), 1067-1081.

(62) Kaniyappan, S., Chandupatla, R. R., Mandelkow, E.-M., and Mandelkow, E. (2017) Extracellular Low-n Oligomers of Tau Cause
Selective Synaptotoxicity without Affecting Cell Viability. Alzheimer's Dementia 13 (11), 1270-1291.

(63) Alonso, d. A. C., Mederlyova, A., Novak, M., Grundke-Iqbal, I., and Iqbal, K. (2004) Promotion of Hyperphosphorylation by Frontotemporal Dementia Tau Mutations. J. Biol. Chem. 279 (33), 34873-34881.

(64) von Bergen, M., Barghorn, S., Li, L., Marx, A., Biernat, J., Mandelkow, E.-M., and Mandelkow, E. (2001) Mutations of Tau Protein in Frontotemporal Dementia Promote Aggregation of Paired Helical Filaments by Enhancing Local $\beta$-Structure. J. Biol. Chem. 276 (51), 48165-48174.

(65) Dawson, H. N., Cantillana, V., Chen, L., and Vitek, M. P. (2007) The Tau N279K Exon 10 Splicing Mutation Recapitulates Frontotemporal Dementia and Parkinsonism Linked to Chromosome 17 Tauopathy in a Mouse Model. J. Neurosci. 27 (34), 9155-9168.

(66) Domoto-Reilly, K., Davis, M. Y., Keene, C. D., and Bird, T. D. (2017) Unusually Long Duration and Delayed Penetrance in a Family with FTD and Mutation in MAPT (V337M). Am. J. Med. Genet., Part B 174 (1), 70-74.

(67) Delisle, M. B., Murrell, J. R., Richardson, R., Trofatter, J. A., Rascol, O., Soulages, X., Mohr, M., Calvas, P., and Ghetti, B. (1999) A Mutation at Codon 279 (N279K) in Exon 10 of the Tau Gene Causes a Tauopathy with Dementia and Supranuclear Palsy. Acta Neuropathol. 98 (1), 62-77.

(68) Hasegawa, M., Smith, M. J., Iijima, M., Tabira, T., and Goedert, M. (1999) FTDP-17 Mutations N279K and S305N in Tau Produce Increased Splicing of Exon 10. FEBS Lett. 443 (2), 93-96.

(69) Tanemura, K., Murayama, M., Akagi, T., Hashikawa, T., Tominaga, T., Ichikawa, M., Yamaguchi, H., and Takashima, A. (2002) Neurodegeneration with Tau Accumulation in a Transgenic Mouse Expressing V337M Human Tau. J. Neurosci. 22 (1), 133-141.

(70) Marshall, C. R., Guerreiro, R., Thust, S., Fletcher, P., Rohrer, J. D., and Fox, N. C. A. (2015) Novel MAPT Mutation Causing Corticobasal Syndrome Led by Progressive Apraxia of Speech. J. Alzheimer's Dis. 48 (4), 923-926.

(71) Momeni, P., Wickremaratchi, M. M., Bell, J., Arnold, R., Beer, R., Hardy, J., Revesz, T., Neal, J. W., and Morris, H. R. (2010) Familial Early Onset Frontotemporal Dementia Caused by a Novel S356T MAPT Mutation, Initially Diagnosed as Schizophrenia. Clin. Neurol. Neurosurg. 112 (10), 917-920.

(72) Cohen, T. J., Friedmann, D., Hwang, A. W., Marmorstein, R., and Lee, V. M. Y. (2013) The Microtubule-Associated Tau Protein Has Intrinsic Acetyltransferase Activity. Nat. Struct. Mol. Biol. 20 (6), $756-762$.

(73) D’Amico, M., Di Carlo, M. G., Groenning, M., Militello, V., Vetri, V., Leone, M., and Thioflavin, T. (2012) Promotes A $\beta(1-40)$ Amyloid Fibrils Formation. J. Phys. Chem. Lett. 3 (12), 1596-1601.

(74) Xu, S., Brunden, K. R., Trojanowski, J. Q., and Lee, V. M.-Y. (2010) Characterization of Tau Fibrillization in Vitro. Alzheimer's Dementia 6 (2), 110-117.

(75) Arimon, M., Sanz, F., Giralt, E., and Carulla, N. (2012) Template-Assisted Lateral Growth of Amyloid-B42 Fibrils Studied by Differential Labeling with Gold Nanoparticles. Bioconjugate Chem. 23 (1), 27-32.

(76) Huseby, C. J., Bundschuh, R., and Kuret, J. (2019) The Role of Annealing and Fragmentation in Human Tau Aggregation Dynamics. J. Biol. Chem. 294 (13), 4728-4737.

(77) Hiraoka, S., Yao, T.-M., Minoura, K., Tomoo, K., Sumida, M., Taniguchi, T., and Ishida, T. (2004) Conformational Transition State Is Responsible for Assembly of Microtubule-Binding Domain of Tau Protein. Biochem. Biophys. Res. Commun. 315 (3), 659-663.

(78) von Bergen, M., Barghorn, S., Biernat, J., Mandelkow, E.-M., and Mandelkow, E. (2005) Tau Aggregation Is Driven by a Transition from Random Coil to Beta Sheet Structure. Biochim. Biophys. Acta, Mol. Basis Dis. 1739 (2-3), 158-166.

(79) Barghorn, S., Biernat, J., and Mandelkow, E. Purification of Recombinant Tau Protein and Preparation of Alzheimer-Paired Helical Filaments In Vitro. In Amyloid Proteins; Methods in Molecular 
BiologyTM; Humana Press: 2005; pp 35-51, DOI: 10.1385/1-59259874-9:035.

(80) Csokova, N., Skrabana, R., Liebig, H.-D., Mederlyova, A., Kontsek, P., and Novak, M. (2004) Rapid Purification of Truncated Tau Proteins: Model Approach to Purification of Functionally Active Fragments of Disordered Proteins, Implication for Neurodegenerative Diseases. Protein Expression Purif. 35 (2), 366-372.

(81) Ikeda, C., Yokota, O., Miki, T., Takenoshita, S., Ishizu, H., Mori, Y., Yamazaki, K., Ozaki, Y., Ueno, S.-I., Ishihara, T., Hasegawa, M., Terada, S., and Yamada, N. (2017) Pick's Disease with Neuronal Four-repeat Tau Accumulation in the Basal Ganglia, Brain Stem Nuclei and Cerebellum. Neuropathology 37 (6), 544-559.

(82) Buée, L., and Delacourte, A. (1999) Comparative Biochemistry of Tau in Progressive Supranuclear Palsy, Corticobasal Degeneration, FTDP-17 and Pick's Disease. Brain Pathol. 9 (4), 681-693.

(83) De Silva, R., Lashley, T., Gibb, G., Hanger, D., Hope, A., Reid, A., Bandopadhyay, R., Utton, M., Strand, C., Jowett, T., Khan, N., Anderton, B., Wood, N., Holton, J., Revesz, T., and Lees, A. (2003) Pathological Inclusion Bodies in Tauopathies Contain Distinct Complements of Tau with Three or Four Microtubule-Binding Repeat Domains as Demonstrated by New Specific Monoclonal Antibodies. Neuropathol. Appl. Neurobiol. 29 (3), 288-302.

(84) Matsumoto, S.-E., Motoi, Y., Ishiguro, K., Tabira, T., Kametani, F., Hasegawa, M., and Hattori, N. (2015) The Twenty-Four KDa CTerminal Tau Fragment Increases with Aging in Tauopathy Mice: Implications of Prion-like Properties. Hum. Mol. Genet. 24 (22), 6403-6416.

(85) Zhou, Y., Shi, J., Chu, D., Hu, W., Guan, Z., Gong, C.-X., Iqbal, K., and Liu, F. (2018) Relevance of Phosphorylation and Truncation of Tau to the Etiopathogenesis of Alzheimer's Disease. Front. Aging Neurosci. 10, 27.

(86) Karikari, T. K., Thomas, R., and Moffat, K. G. (2020) The C291R Tau Variant Forms Different Types of Protofibrils. Front. Mol. Neurosci. 13, 39.

(87) Ansari, A. M., Ahmed, A. K., Matsangos, A. E., Lay, F., Born, L. J., Marti, G., Harmon, J. W., and Sun, Z. (2016) Cellular GFP Toxicity and Immunogenicity: Potential Confounders in in Vivo Cell Tracking Experiments. Stem Cell Rev. 12 (5), 553-559.

(88) Ganini, D., Leinisch, F., Kumar, A., Jiang, J., Tokar, E. J., Malone, C. C., Petrovich, R. M., and Mason, R. P. (2017) Fluorescent Proteins Such as EGFP Lead to Catalytic Oxidative Stress in Cells. Redox Biol. 12, 462-468.

(89) Hill, E., Karikari, T. K., Moffat, K. G., Richardson, M. J. E., and Wall, M. J. (2019) Introduction of Tau Oligomers into Cortical Neurons Alters Action Potential Dynamics and Disrupts Synaptic Transmission and Plasticity. eNeuro 6 (5), ENEURO.0166-19.2019.

(90) Hill, E., Wall, M. J., Moffat, K. G., and Karikari, T. K. (2020) Understanding the Pathophysiological Actions of Tau Oligomers: A Critical Review of Current Electrophysiological Approaches. Front. Mol. Neurosci. 13, 155.

(91) Falcon, B., Cavallini, A., Angers, R., Glover, S., Murray, T. K., Barnham, L., Jackson, S., O’Neill, M. J., Isaacs, A. M., Hutton, M. L., Szekeres, P. G., Goedert, M., and Bose, S. (2015) Conformation Determines the Seeding Potencies of Native and Recombinant Tau Aggregates. J. Biol. Chem. 290 (2), 1049-1065.

(92) Sahara, N., Maeda, S., Murayama, M., Suzuki, T., Dohmae, N., Yen, S.-H., and Takashima, A. (2007) Assembly of Two Distinct Dimers and Higher-Order Oligomers from Full-Length Tau. Eur. J. Neurosci. 25 (10), 3020-3029.

(93) Yamin, G., Coppola, G., and Teplow, D. B. (2016) Design Characterization, and Use of a Novel Amyloid $\beta$-Protein Control for Assembly, Neurotoxicity, and Gene Expression Studies. Biochemistry 55 (36), 5049-5060.

(94) Vadukul, D. M., Gbajumo, O., Marshall, K. E., and Serpell, L. C. (2017) Amyloidogenicity and Toxicity of the Reverse and Scrambled Variants of Amyloid- $\beta$ 1-42. FEBS Lett. 591 (5), 822-830.

(95) Goedert, M., and Jakes, R. (2005) Mutations Causing Neurodegenerative Tauopathies. Biochim. Biophys. Acta, Mol. Basis Dis. 1739 (2-3), 240-250.
(96) Goedert, M. (2005) Tau Gene Mutations and Their Effects. Mov. Disord. 20 (S12), S45-S52. 